\title{
On The Modified Burr XII-Power Distribution: Development, Properties, Characterizations and Applications
}

Fiaz Ahmad Bhatti

National College of Business Administration and Economics, Lahore Pakistan. fiazahmad72@gmail.com

G.G. Hamedani

Marquette University, Milwaukee, WI 53201-1881, USA

g.hamedani@mu.edu

Mustafa Ç. Korkmaz

Department of Measurement and Evaluation, Artvin Çoruh University, Artvin, Turkey

mcagatay@artvin.edu.tr

Munir Ahmad

National College of Business Administration and Economics, Lahore Pakistan.

munirahmaddr@yahoo.co.uk

\begin{abstract}
In this paper, a flexible lifetime distribution with increasing, decreasing and bathtub hazard rate called the Modified Burr XII-Power (MBXII-Power) is developed on the basis of the T-X family technique. The density function of the MBXII-Power is arc, exponential, left-skewed, right-skewed, J, reverse-J and symmetrical shaped. Descriptive measures such as moments, moments of order statistics, incomplete moments, inequality measures, residual life functions and reliability measures are theoretically established. The MBXII-Power distribution is characterized via different techniques. Parameters of the MBXII-Power distribution are estimated using maximum likelihood method. The simulation study is performed on the basis of graphical results to see the performance of maximum likelihood estimates (MLEs) of the MBXIIPower distribution. The potentiality of the MBXII-Power distribution is demonstrated by its application to real data sets: survival times of pigs, survival times of patients and quarterly earnings.
\end{abstract}

Key Words: Moments, Reliability, Characterizations, Maximum Likelihood Estimation.

\section{Introduction}

A flexible model for the analysis of lifetime data sets is often always attractive to the researchers. Pareto, power function, exponential and Weibull distribution are of interest due to the attractive flexibility and simplicity.

In recent decades, many continuous univariate distributions have been developed but various data sets from reliability, insurance, finance, climatology, biomedical sciences and other areas do not follow these distributions. Therefore, modified, extended and generalized distributions and their applications to problems in these areas is a clear need of day.

The modified, extended and generalized distributions are obtained by the introduction of some transformation or addition of one or more parameters to the baseline distribution. 
These new developed distributions provide better fit to data than the sub and competing models.

Burr (1942) suggested 12 distributions as Burr family to fit cumulative frequency functions on frequency data. Burr distributions XII, III and X are frequently used. BurrXII (BXII) distribution has wide applications in modeling insurance data in finance and business and failure time data in reliability, survival and acceptance sampling plans.

Many modified, extended and generalized forms of BXII distribution are available in literature such as Burr (Takahasi;1965), extended three-parameter BXII (Shao et al.; 2004), generalized BXII (Olapade;2008), beta BXII (Paranaíba et al.; 2011), Kumaraswamy BXII (Paranaíba et al.; 2013), BXII power series (Silver and Cordeiro ;2015), McDonald BXII (Gomes et al.; 2015), generalized BXII-Poisson (Muhammad; 2016), extended BXII (Ghosh and Bourguignon; 2017), BXII modified Weibull (Mdlongwa et al.;2017), gamma BXII (Guerra et al.; 2017), BXII modified Weibull (Mdlongwa et al.; 2017), five-parameter BXII (Mead and Afify; 2017), new BXII (Yari and Tondpour; 2017), four-parameter BXII (Afify et al.; 2018), generalized log BXII(Bhatti et al.;2018a) and modified log BXII distribution (Bhatti et al.;2018b).

The main goal of this paper is to obtain a more flexible distribution for the lifetime applications called the MBXII-Power distribution. The MBXII-Power density is arc, exponential, left-skewed, right-skewed, J, reverse-J and symmetrical shaped. The MBXII-Power distribution has increasing, decreasing, decreasing-increasing, inverted bathtub and bathtub hazard rate function. The MBXII-Power distribution is the best model for modeling real data in survival analysis, life testing, reliability, economics and other areas of research. The MBXII-Power distribution offers better fits than nested and competing models.

This paper is sketched into the following sections. In Section 2, the MBXII-Power distribution is derived on the basis of the T-X family technique, transformation and compounding mixture of distributions. The MBXII-Power distribution is also studied in terms of basic structural properties, sub-models, and some plots. In Section 3, moments, incomplete moments, inequality measures, residual and reverse residual life function and some other properties are theoretically derived. In Section 4, stress-strength reliability and multicomponent stress-strength reliability of the model are studied. In Section 5, MBXII-Power distribution is characterized via (i) conditional expectation; (ii) truncated moment; (iii) hazard function; (iv) Mills ratio; (v) certain functions of the random variable and (vi) conditional expectation of record values. In Section 6, the parameters of the MBXII-Power are estimated using maximum likelihood method. In Section 7, a simulation study is performed on the basis of graphical results by using the MBXIIPower distribution to see the performance of MLEs corresponding to this distribution. In Section 8, the potentiality of the MBXII-Power distribution is demonstrated by its application to real data sets: survival times of pigs, survival times of patients and quarterly earnings. Goodness of fit of the probability distribution through different methods is studied. The concluding remarks are given in Section 9. 


\section{DEVELOPMENT OF THE MBXII-Power DISTRIBUTION}

The probability density function (pdf) and cumulative distribution function (cdf) of the power distribution is given, respectively, by

$$
g(x)=\kappa \lambda^{-\kappa} x^{\kappa-1}, \kappa>0, \lambda>0,0<x<\lambda,
$$

and

$$
G(x)=\left(\frac{x}{\lambda}\right)^{\kappa}=\frac{x^{\kappa}}{\lambda^{\kappa}} \quad, \kappa>0, \lambda>0,0 \leq x<\lambda .
$$

The odds ratio for the power random variable $\mathrm{X}$ is

$$
W[G(x)]=\frac{G(x)}{\bar{G}(x)}=\frac{x^{\kappa}}{\lambda^{\kappa}-x^{\kappa}} .
$$

Gurvich et al. (1997) replaced " $x$ " with odds ratio in the Weibull distribution for the development of a class of extended Weibull distributions. Alzaatreh et al. (2013) developed the cdf of the T-X family of distributions as

$$
F(x)=\int_{a}^{W[G(x)]} r(t) d t
$$

where $\mathrm{W}(\mathrm{G}(\mathrm{x}))$ is function of $\mathrm{G}(\mathrm{x})$ and $\mathrm{r}(\mathrm{t})$ is the pdf of a non-negative random variable.

Bourguignon et al. (2014) inserted the odds ratio of a baseline distribution in place of ' $x$ ' in the cdf of the Weibull distribution for the development of a new family of distributions.

The MBXII-Power is developed by inserting the odds ratio of the power random in place of ' $x$ ' in the cdf of MBXII distribution. The cdf for the MBXII-Power distribution is obtained as

$$
\begin{gathered}
F(x)=\int_{0}^{\left(\frac{x^{\kappa}}{\lambda^{\kappa}-x^{\kappa}}\right)} \alpha \beta t^{\beta-1}\left(1+\gamma t^{\beta}\right)^{-\frac{\alpha}{\gamma}-1} d t, \\
F(x)=1-\left[1+\gamma\left(\frac{x^{\kappa}}{\lambda^{\kappa}-x^{\kappa}}\right)^{\beta}\right]^{-\frac{\alpha}{\gamma}}, 0 \leq x \leq \lambda .
\end{gathered}
$$

The pdf of the MBXII-Power distribution is

$$
f(x)=\frac{\alpha \beta \kappa \lambda^{\kappa} x^{\kappa \beta-1}}{\left(\lambda^{\kappa}-x^{\kappa}\right)^{\beta+1}}\left[1+\gamma\left(\frac{x^{\kappa}}{\lambda^{\kappa}-x^{\kappa}}\right)^{\beta}\right]^{-\frac{\alpha}{\gamma}-1}, 0<x<\lambda,
$$

where $\alpha>0, \beta>0, \gamma>0, \kappa>0$ and $\lambda>0$ are parameters.

\subsection{Transformations and Compounding}

The MBXII-Power distribution is derived through (i) ratio of exponential and gamma random variables and (ii) compounding generalized Weibull Power (GW-P) and gamma distributions. 
Lemma. (i) If $Z_{1} \sim \exp$.(1) and $Z_{2} \sim \operatorname{Gamma}(\alpha / \gamma, 1)$, then for $Z_{1}=\gamma\left(\frac{X^{\kappa}}{\lambda^{\kappa}-X^{\kappa}}\right)^{\beta} Z_{2}$, we have

$$
X=\lambda\left[1+\left(\gamma Z_{2}\left(Z_{1}\right)^{-1}\right)^{\frac{1}{\beta}}\right]^{-\frac{1}{\kappa}} \sim \text { MBXII }-\operatorname{Power}(\alpha, \beta, \gamma, \kappa, \lambda) .
$$

ii) If $Y / \beta, \kappa, \gamma, \lambda, \theta \sim G W-P(y ; \beta, \kappa, \gamma, \lambda, \theta)$ and $\theta / \alpha, \gamma \sim \operatorname{facrtional~gamma}(\theta ; \alpha, \gamma)$, then integrating the effect of $\theta$ with the help of

$$
f(y, \alpha, \beta, \gamma, \lambda)=\int_{0}^{\infty} g(y / \beta, \kappa, \gamma, \lambda / \theta) g(\theta / \alpha, \gamma) d \theta,
$$

we have $Y \sim M B X I I-\operatorname{Power}(\alpha, \beta, \gamma, \kappa, \lambda)$.

\subsection{Basic Structural Properties}

The survival, hazard, cumulative hazard, reverse hazard, elasticity functions and the Mills ratio of a random variable $\mathrm{X}$ with the MBXII-Power distribution are given, respectively, by

$$
\begin{aligned}
& S(x)=\left[1+\gamma\left(\frac{x^{\kappa}}{\lambda^{\kappa}-x^{\kappa}}\right)^{\beta}\right]^{-\frac{\alpha}{\gamma}}, 0 \leq x \leq \lambda, \\
& h(x)=\frac{\alpha \beta \kappa \lambda^{\kappa} x^{\kappa \beta-1}}{\left(\lambda^{\kappa}-x^{\kappa}\right)^{\beta+1}}\left[1+\gamma\left(\frac{x^{\kappa}}{\lambda^{\kappa}-x^{\kappa}}\right)^{\beta}\right]^{-1}, 0<x<\lambda, \\
& r(x)=\frac{d}{d x} \ln \left\{1-\left[1+\gamma\left(\frac{x^{\kappa}}{\lambda^{\kappa}-x^{\kappa}}\right)^{\beta}\right]^{-\frac{\alpha}{\gamma}}\right\}, 0<x<\lambda \\
& H(x)=\frac{\alpha}{\gamma} \ln \left[1+\gamma\left(\frac{x^{\kappa}}{\lambda^{\kappa}-x^{\kappa}}\right)^{\beta}\right], 0<x<\lambda, \\
& e(x)=\frac{d}{d \ln x} \ln \left\{1-\left[1+\gamma\left(\frac{x^{\kappa}}{\lambda^{\kappa}-x^{\kappa}}\right)^{\beta}\right]^{-\frac{\alpha}{\gamma}}\right\},
\end{aligned}
$$

and

$$
m(x)=\frac{\left(\lambda^{\kappa}-x^{\kappa}\right)^{\beta+1}}{\alpha \beta \kappa \lambda^{\kappa} x^{\kappa \beta-1}}\left[1+\gamma\left(\frac{x^{\kappa}}{\lambda^{\kappa}-x^{\kappa}}\right)^{\beta}\right]
$$


The quantile function of the MBXII-Power distribution is

$$
x_{q}=\lambda\left[\left[\gamma^{-1}\left((1-q)^{-\frac{\gamma}{\alpha}}-1\right)\right]^{-\frac{1}{\beta}}+1\right]^{-\frac{1}{\kappa}} \text {. }
$$

The MBXII-Power random number generator is

$$
X=\lambda\left[\left[\gamma^{-1}\left((1-Z)^{-\frac{\gamma}{\alpha}}-1\right)\right]^{-\frac{1}{\beta}}+1\right]^{-\frac{1}{\kappa}}
$$

where the random variable $\mathrm{Z}$ has the uniform distribution on $(0,1)$.

\subsection{Sub-Models}

The MBXII-Power distribution has the following sub models.

Table 1: Sub-Models of MBXII-Power Distribution

\begin{tabular}{|l|l|l|l|l|l|l|}
\hline Sr. No. & $\alpha$ & $\beta$ & $\gamma$ & $\kappa$ & $\lambda$ & Name of distribution \\
\hline 1 & $\alpha$ & $\beta$ & $\gamma$ & $\kappa$ & $\lambda$ & MBXII-Power distribution \\
\hline 2 & $\alpha$ & $\beta$ & 1 & $\kappa$ & $\lambda$ & BXII-Power distribution \\
\hline 3 & $\alpha$ & 1 & 1 & $\kappa$ & $\lambda$ & Lomax-Power distribution \\
\hline 4 & 1 & $\beta$ & 1 & $\kappa$ & $\lambda$ & $\begin{array}{l}\text { Log-logistic-Power } \\
\text { distribution }\end{array}$ \\
\hline 5 & $\alpha$ & $\beta$ & $\gamma \rightarrow 0$ & $\kappa$ & $\lambda$ & $\begin{array}{l}\text { Generalized Weibull-Power } \\
\text { distribution }\end{array}$ \\
\hline 6 & 1 & $\beta$ & $\gamma \rightarrow 0$ & $\kappa$ & $\lambda$ & $\begin{array}{l}\text { Weibull-Power distribution } \\
\text { (Tahir et al. ;2014) }\end{array}$ \\
\hline
\end{tabular}

\subsection{Shapes of the MBXII-Power Density and Hazard Rate Functions}

The following graphs show that shapes of the MBXII-Power density are arc, left-skewed, right-skewed, J, reverse-J, exponential and symmetrical (Fig. 1). The MBXII- Power distribution has increasing, decreasing, decreasing-increasing, inverted bathtub and bathtub hazard rate function (Fig. 2 and Fig. 3). 


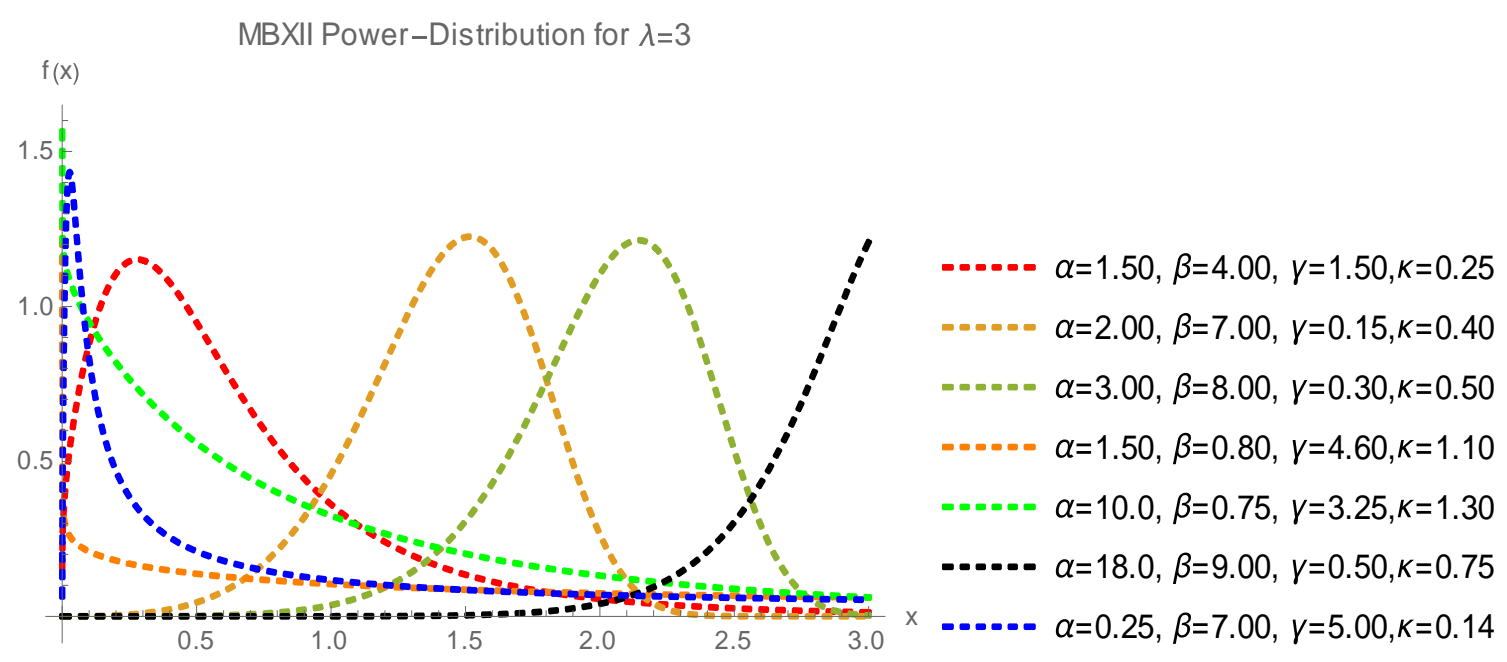

Fig. 1: Plot of pdf of the MBXII-Power Distribution

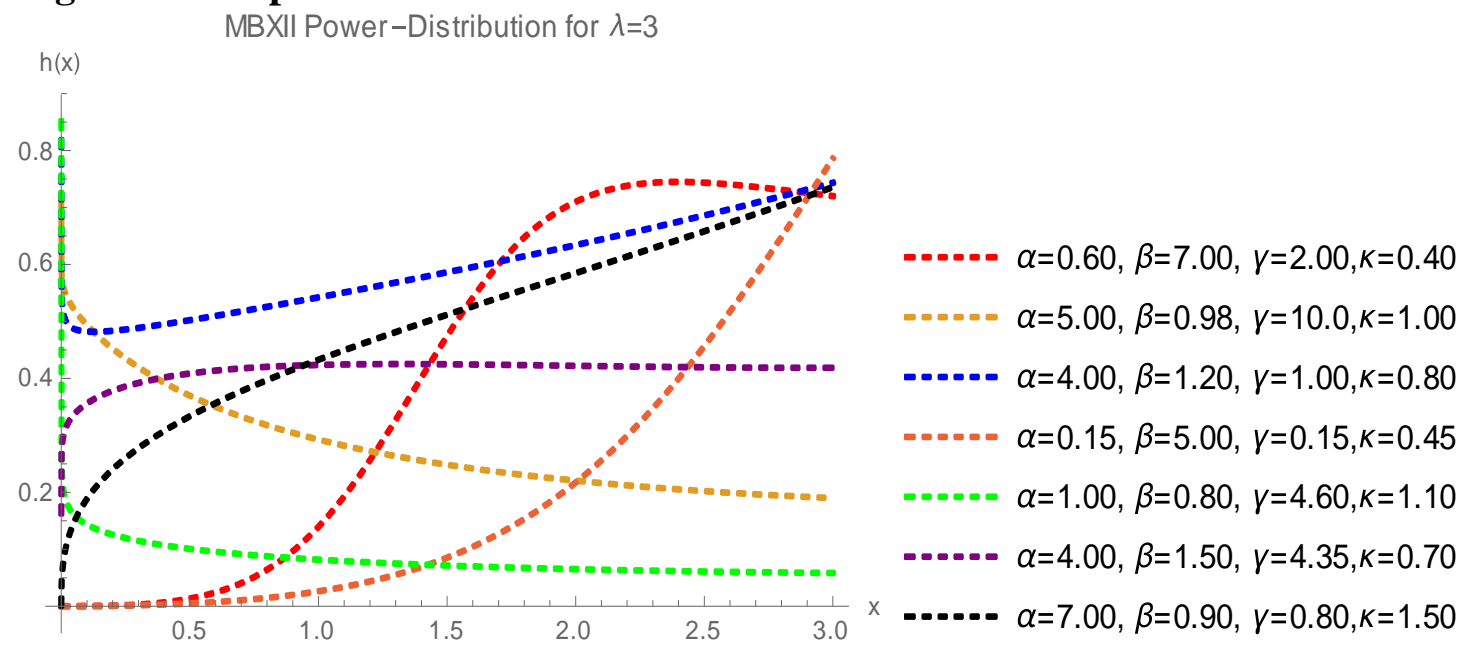

Fig.2: Plot of hrf of the MBXII-Power Distribution

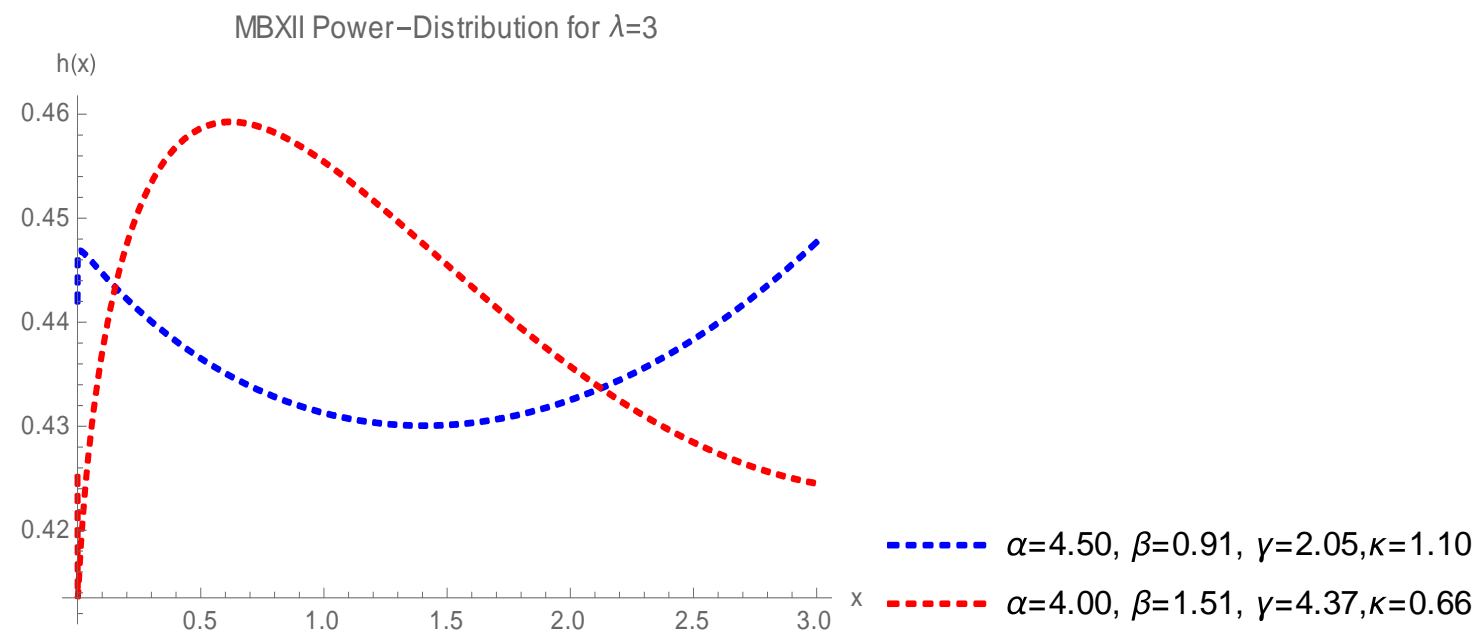

Fig. 3: Plot of hrf of the MBXII-Power Distribution 


\section{MOMENTS}

Moments, incomplete moments, inequality measures, residual and reverse residual life function and some other properties are theoretically derived in this section.

\subsection{Moments about Origin}

The $r^{\text {th }}$ ordinary moment for the MBXII-Power distribution is,

$$
\begin{gathered}
E\left(X^{r}\right)=\int_{0}^{\infty} x^{r} f(x) d x, \\
E\left(X^{r}\right)=\int_{0}^{\lambda} x^{r} \frac{\alpha \beta \kappa \lambda^{\kappa} x^{\kappa \beta-1}}{\left(\lambda^{\kappa}-x^{\kappa}\right)^{\beta+1}}\left[1+\gamma\left(\frac{x^{\kappa}}{\lambda^{\kappa}-x^{\kappa}}\right)^{\beta}\right]^{-\frac{\alpha}{\gamma}-1} d x .
\end{gathered}
$$

Letting $\gamma\left(\frac{x^{\kappa}}{\lambda^{\kappa}-x^{\kappa}}\right)^{\beta}=w$, we obtain

$$
\begin{aligned}
& E\left(X^{r}\right)=\alpha \lambda^{r} \sum_{\ell=0}^{\infty} \frac{(-1)^{\ell}}{\ell !}\left(\frac{r}{\kappa}\right)_{\ell} \gamma^{\frac{\ell}{\beta}} \int_{0}^{\infty} w^{1-\frac{\ell}{\beta}-1}[1+w]^{-\frac{\alpha}{\gamma}-1} d x, \\
& E\left(X^{r}\right)=\alpha \lambda^{r} \sum_{\ell=0}^{\infty}\left(\frac{r}{\kappa}\right)_{\ell} \frac{(-1)^{\ell}}{\ell !} \gamma^{\frac{\ell}{\beta}} B\left(1-\frac{\ell}{\beta}, \frac{\alpha}{\gamma}+\frac{\ell}{\beta}\right) .
\end{aligned}
$$

The factorial moments of the MBXII-Power distribution are given by

$$
E[X]_{n}=\sum_{r=1}^{n} \varphi_{r} E\left(X^{r}\right)=\alpha \lambda^{r} \sum_{r=1}^{m} \varphi_{r} \sum_{\ell=0}^{\infty} \frac{(-1)^{\ell}}{\ell !}\left(\frac{r}{\kappa}\right)_{\ell} \gamma^{\frac{\ell}{\beta}} B\left(1-\frac{\ell}{\beta}, \frac{\alpha}{\gamma}+\frac{\ell}{\beta}\right),
$$

where $[X]_{i}=X(X+1)(X+2) \ldots \ldots(X+i-1)$ and $\varphi_{r}$ is the Stirling number of the first kind.

The Mellin transform of $\mathrm{X}$ with the MBXII-Power distribution is

$$
\begin{gathered}
\mathrm{M}\{f(x) ; \mathrm{s}\}=\int_{0}^{\lambda} x^{s-1} \frac{\alpha \beta \kappa \lambda^{\kappa} x^{\kappa \beta-1}}{\left(\lambda^{\kappa}-x^{\kappa}\right)^{\beta+1}}\left[1+\gamma\left(\frac{x^{\kappa}}{\lambda^{\kappa}-x^{\kappa}}\right)^{\beta}\right]^{-\frac{\alpha}{\gamma}-1} d x, \\
M\{f(x) ; \mathrm{s}\}=E\left[X^{s-1}\right]=\alpha \lambda^{(s-1)} \sum_{\ell=0}^{\infty} \frac{(-1)^{\ell}}{\ell !}\left(\frac{s-1}{\kappa}\right)_{\ell} \gamma^{\frac{\ell}{\beta}} B\left(1-\frac{\ell}{\beta}, \frac{\alpha}{\gamma}+\frac{\ell}{\beta}\right) .
\end{gathered}
$$

The $\mathrm{r}^{\text {th }}$ moment about means, Pearson's measures for skewness $\gamma_{1}$ and kurtosis $\beta_{2}$, moment generating function and cumulants of $\mathrm{X}$ for the MBXII-Power distribution are achieved from the relations

$$
\mu_{r}=\sum_{i=1}^{r}\left(\begin{array}{l}
r \\
i
\end{array}\right)(-1)^{i} \mu_{i}^{\prime} \mu_{i-r}^{\prime}, \gamma_{1}=\frac{\mu_{3}}{\left(\mu_{2}\right)^{\frac{3}{2}}}, \beta_{2}=\frac{\mu_{4}}{\left(\mu_{2}\right)^{2}}, M_{X}(t)=E\left[e^{t X}\right]=\sum_{r=1}^{\infty} \frac{t^{r}}{\mathrm{r} !} E(X)^{r},
$$

and $k_{r}=\mu_{r}^{\prime}-\sum_{c=1}^{r-1}\left(\begin{array}{l}r-1 \\ c-1\end{array}\right) k_{c} \mu_{r-c}^{\prime}$ 
Bhatti, F.A., Hamedani, G. G. Korkmaz, M. C. and Ahmad M,

Table 2, shows the numerical measure of the median, mean, standard deviation, skewness and Kurtosis of the MBXII-Power distribution for selected parameter values to describe their effect on these measures.

Table 2: Median, mean, standard deviation, skewness and Kurtosis of the MBXIIPower Distribution

\begin{tabular}{|l|r|r|r|r|r|}
\hline $\begin{array}{l}\text { Parameters } \\
\alpha, \beta, \gamma, \kappa, \lambda=3\end{array}$ & Median & \multicolumn{1}{l|}{ Mean } & $\begin{array}{l}\text { Standard } \\
\text { Deviation }\end{array}$ & Skewness & Kurtosis \\
\hline $0.5,1.5,0.5,0.5$ & 3.7631 & 3.9857 & 2.5178 & 0.3076 & 2.1207 \\
\hline $0.3,2,0.5,0.5$ & 4.5667 & 4.6996 & 2.4496 & 0.1596 & 2.1314 \\
\hline $0.5,0.5,0.5,0.5$ & 6.3956 & 5.4553 & 3.9384 & -0.23 & 1.3826 \\
\hline $1,0.5,0.5,0.5$ & 1.6543 & 3.248 & 3.4893 & 0.6646 & 1.892 \\
\hline $1,1,0.5,0.5$ & 2.0502 & 2.7395 & 2.4807 & 0.7915 & 2.59 \\
\hline $1,1,1,0.5$ & 2.4965 & 3.3306 & 2.9797 & 0.6399 & 2.1452 \\
\hline $1,1,1,1$ & 4.9918 & 4.9949 & 2.8853 & 0.0019 & 1.8006 \\
\hline $1,3,1,1$ & 4.9973 & 4.9975 & 1.3663 & 0.0012 & 2.9944 \\
\hline $0.5,2,0.5,0.5$ & 3.4317 & 3.636 & 2.0682 & 0.4289 & 2.5452 \\
\hline $0.5,3,0.5,0.5$ & 3.1078 & 3.2442 & 1.4872 & 0.5305 & 3.2039 \\
\hline $3,3,3,0.5$ & 1.6764 & 1.9096 & 1.1933 & 1.2566 & 5.2679 \\
\hline $3,3,3,3$ & 7.4241 & 7.3708 & 0.8359 & -0.4701 & 3.7062 \\
\hline $5,5,1,5$ & 8.3492 & 8.3044 & 0.2796 & -1.1778 & 5.7567 \\
\hline $2,3,3,1.5$ & 5.9485 & 5.9738 & 1.3597 & 0.0082 & 2.9399 \\
\hline $0.75,7,1.75,5$ & 8.8065 & 8.8227 & 0.3009 & 0.1366 & 3.5612 \\
\hline $5,0.5,0.3,1$ & 0.1955 & 0.657 & 1.0814 & 2.8139 & 12.4295 \\
\hline $5,0.5,5,0.5$ & 0.0148 & 1.2772 & 2.6524 & 2.1994 & 6.542 \\
\hline $3.5,0.5,5,0.3$ & 0.0198 & 2.0844 & 3.4417 & 1.403 & 3.3114 \\
\hline $1.5,0.5,5,0.5$ & 5.2618 & 5.0389 & 4.396 & -0.0222 & 1.1774 \\
\hline $0.5,0.5,5,0.5$ & 9.9963 & 7.76 & 3.7576 & -1.3264 & 2.9652 \\
\hline
\end{tabular}

\subsection{Moments of Order Statistics}

Moments of order statistics have applications in reliability and life testing. Moments of order statistics are also designed for replacement policy to predict of failure of future items obtained from few initial failures.

The pdf of the $m t h$ order statistic $X_{\mathrm{m}: n}$ is

$$
f\left(x_{\mathrm{m}: n}\right)=\frac{1}{B(\mathrm{~m}, n-m+1)}[F(x)]^{m-1}[1-F(x)]^{n-m} f(x) .
$$

The pdf of $m t h$ order statistic $X_{\mathrm{m}: n}$ for the MBXII-Power distribution is

$$
f\left(x_{\mathrm{m}: n}\right)=\frac{1}{B(\mathrm{~m}, n-m+1)} \sum_{i=0}^{m-1}(-1)^{i}\left(\begin{array}{l}
m-1 \\
i
\end{array}\right) \frac{\alpha \beta \kappa \lambda^{\kappa} x^{\kappa \beta-1}}{\left(\lambda^{\kappa}-x^{\kappa}\right)^{\beta+1}}\left[1+\gamma\left(\frac{x^{\kappa}}{\lambda^{\kappa}-x^{\kappa}}\right)^{\beta}\right]^{-\frac{\alpha}{\gamma}(n-m+1+i)-1} .
$$


Moments about the origin of $m t h$ order statistic $X_{\mathrm{m}: n}$ for the MBXII-Power distribution are

$$
\begin{gathered}
E\left(X_{\mathrm{m}: n}^{r}\right)=\int_{0}^{\lambda} x^{r} \frac{1}{B(\mathrm{~m}, n-m+1)} \sum_{i=0}^{m-1}(-1)^{i}\left(\begin{array}{l}
m-1 \\
i
\end{array}\right) \frac{\alpha \beta \kappa \lambda^{\kappa} x^{\kappa \beta-1}}{\left(\lambda^{\kappa}-x^{\kappa}\right)^{\beta+1}}\left[1+\gamma\left(\frac{x^{\kappa}}{\lambda^{\kappa}-x^{\kappa}}\right)^{\beta}\right]^{-\frac{\alpha}{\gamma}(n-m+1+i)-1} d x \\
E\left(X_{\mathrm{m}: n}^{r}\right)=\frac{\alpha \lambda^{r}}{B(\mathrm{~m}, n-m+1)} \sum_{i=0}^{m-1} \sum_{\ell=0}^{\infty} \frac{(-1)^{i+\ell} \gamma^{\frac{\ell}{\beta}}}{\ell !}\left(\begin{array}{c}
m-1 \\
i
\end{array}\right)\left(\frac{r}{\kappa}\right)_{\ell} \times \\
B\left[1-\frac{\ell}{\beta}, \frac{\alpha}{\gamma}(n-m+1+i)+\frac{\ell}{\beta}\right], \mathrm{r}=1,2,3, \ldots
\end{gathered}
$$

\subsection{Incomplete Moments}

Incomplete moments are used in mean inactivity life, mean residual life function, and other inequality measures.

The $s^{\text {th }}$ incomplete moment for the MBXII-Power distribution is

$$
\begin{gathered}
M_{s}^{\prime}(z)=\int_{0}^{z} x^{s} \frac{\alpha \beta \kappa \lambda^{\kappa} x^{\kappa \beta-1}}{\left(\lambda^{\kappa}-x^{\kappa}\right)^{\beta+1}}\left[1+\gamma\left(\frac{x^{\kappa}}{\lambda^{\kappa}-x^{\kappa}}\right)^{\beta}\right]^{-\frac{\alpha}{\gamma}-1} d x, \\
M_{s}^{\prime}(z)=\alpha \lambda^{s} \sum_{\ell=0}^{\infty} \frac{(-1)^{\ell}}{\ell !}\left(\frac{s}{\kappa}\right)_{\ell} \gamma^{\frac{\ell}{\beta}} B_{w(z)}\left(1-\frac{\ell}{\beta}, \frac{\alpha}{\gamma}+\frac{\ell}{\beta}\right),
\end{gathered}
$$

where $w(z)=\gamma\left(\frac{z^{\kappa}}{\lambda^{\kappa}-z^{\kappa}}\right)^{\beta}$ and $B_{z}(.,$.$) is incomplete beta function.$

The mean deviation about mean is $M D_{\bar{X}}=E\left|X-\mu_{1}^{1}\right|=2 \mu_{1}^{1} F\left(\mu_{1}^{1}\right)-2 \mu_{1}^{1} M_{1}^{\prime}\left(\mu_{1}^{1}\right)$ and mean deviation about median is $M D_{M}=E|X-M|=2 M F(M)-2 M M_{1}^{\prime}(M) \quad$ where $\mu_{1}^{\prime}=E(X)$, and $M=Q_{\frac{1}{2}}$. Bonferroni and Lorenz curves for a specified probability $\mathrm{p}$ are computed as by $\mathrm{B}(p)=M_{1}^{\prime}(q) / p \mu_{1}^{1}$ and $L(p)=M_{1}^{\prime}(q) / \mu_{1}^{1}$ where $q=Q(p)$.

\subsection{Residual Life Functions}

The residual life says $m_{n}(z)$ of $\mathrm{X}$ for the MBXII-Power distribution has the following $\mathrm{n}^{\text {th }}$ moment

$$
\begin{gathered}
m_{n}(z)=E\left[(X-z)^{n} \mid X>z\right]=\frac{1}{S(z)} \int_{z}^{\lambda}(x-z)^{s} f(x) d x, \\
m_{n}(z)=\frac{1}{S(z)} \sum_{s=0}^{n}\left(\begin{array}{c}
n \\
s
\end{array}\right)(-z)^{n-s} E_{X>z}\left(X^{s}\right) \\
m_{n}(z)=\frac{1}{\bar{F}(z)} \sum_{s=0}^{n} \sum_{\ell=0}^{\infty} \frac{(-1)^{\ell}}{\ell !}\left(\begin{array}{l}
n \\
s
\end{array}\right)(-z)^{n-s}\left(\frac{s}{\kappa}\right)_{\ell} \gamma^{\frac{\ell}{\beta}} \alpha \lambda^{s}\left[B\left(1-\frac{\ell}{\beta}, \frac{\alpha}{\gamma}+\frac{\ell}{\beta}\right)-B_{w(z)}\left(1-\frac{\ell}{\beta}, \frac{\alpha}{\gamma}+\frac{\ell}{\beta}\right)\right] .
\end{gathered}
$$

The average remaining lifetime of a component at time, z say $m_{1}(z)$, or life expectancy called mean residual life (MRL) function is 


$$
m_{1}(z)=\frac{1}{\bar{F}(z)} \sum_{s=0}^{1} \sum_{\ell=0}^{\infty} \frac{(-1)^{\ell}}{\ell !}\left(\begin{array}{l}
1 \\
s
\end{array}\right)(-z)^{1-s}\left(\frac{s}{\kappa}\right)_{\ell} \gamma^{\frac{\ell}{\beta}} \alpha \lambda^{s}\left[B\left(1-\frac{\ell}{\beta}, \frac{\alpha}{\gamma}+\frac{\ell}{\beta}\right)-B_{w(z)}\left(1-\frac{\ell}{\beta}, \frac{\alpha}{\gamma}+\frac{\ell}{\beta}\right)\right] .
$$

The reverse residual life, say $M_{n}(z)$, of X for the MBXII-Power distribution is

$$
\begin{gathered}
M_{n}(z)=E\left[(z-X)^{n} / X \leq z\right]=\frac{1}{F(z)} \int_{0}^{z}(z-x)^{n} f(x) d x, \\
M_{n}(z)=\frac{1}{F(z)} \sum_{s=0}^{n}(-1)^{s}\left(\begin{array}{c}
n \\
s
\end{array}\right) z^{n-s} E_{X \leq z}\left(X^{s}\right), \\
M_{n}(z)=\frac{1}{F(z)} \sum_{s=0}^{n}(-1)^{s}\left(\begin{array}{l}
n \\
s
\end{array}\right) z^{n-s} \alpha \lambda^{s} \sum_{\ell=0}^{\infty} \frac{(-1)^{\ell}}{\ell !}\left(\frac{s}{\kappa}\right)_{\ell} \gamma^{\frac{\ell}{\beta}} B_{w(z)}\left(1-\frac{\ell}{\beta}, \frac{\alpha}{\gamma}+\frac{\ell}{\beta}\right) .
\end{gathered}
$$

The waiting time $\mathrm{z}$ for failure of a component has passed with condition that this failure had happened in the interval $[0, \mathrm{z}]$ is called mean waiting time (MWT) or mean inactivity time.

The waiting time $\mathrm{z}$ for failure of a component for the MBXII-Power distribution is

$$
M_{1}(z)=\frac{1}{F(z)} \sum_{s=0}^{1}(-1)^{s}\left(\begin{array}{l}
1 \\
s
\end{array}\right) z^{1-s} \alpha \lambda^{s} \sum_{\ell=0}^{\infty} \frac{(-1)^{\ell}}{\ell !}\left(\frac{s}{\kappa}\right)_{\ell} \gamma^{\frac{\ell}{\beta}} B_{w(z)}\left(1-\frac{\ell}{\beta}, \frac{\alpha}{\gamma}+\frac{\ell}{\beta}\right) .
$$

\section{RELIABILITY MEASURES}

In this section, different reliability measures for the MBXII-Power distribution are studied.

\subsection{Stress-Strength Reliability of the MBXII-Power Distribution}

Let $\quad X_{1} \sim M B X I I-P o w e r\left(\alpha_{1}, \beta, \gamma, \kappa, \lambda\right), X_{2} \sim M B X I I-\operatorname{Power}\left(\alpha_{2}, \beta, \gamma, \kappa, \lambda\right)$ and $X_{1}$ represents strength and $X_{2}$ represents stress. Then the reliability of the component is:

$$
\begin{gathered}
\mathrm{R}=\operatorname{Pr}\left(\mathrm{X}_{2}<X_{1}\right)=\int_{-\infty}^{\infty} \int_{-\infty}^{x_{1}} f\left(x_{1}, x_{2}\right) d x_{2} d x_{1}=\int_{0}^{\infty} f_{x_{1}}(x) F_{x_{2}}(x) d x \\
R=\int_{0}^{\lambda} \frac{\alpha_{1} \beta \kappa \lambda^{\kappa} x^{\kappa \beta-1}}{\left(\lambda^{\kappa}-x^{\kappa}\right)^{\beta+1}}\left[1+\gamma\left(\frac{x^{\kappa}}{\lambda^{\kappa}-x^{\kappa}}\right)^{\beta}\right]^{-\frac{\alpha_{1}}{\gamma}-1}\left\{1-\left[1+\gamma\left(\frac{x^{\kappa}}{\lambda^{\kappa}-x^{\kappa}}\right)^{\beta}\right]^{-\frac{\alpha_{2}}{\gamma}}\right\} d x=\frac{\alpha_{2}}{\left(\alpha_{1}+\alpha_{2}\right)} .
\end{gathered}
$$

$\mathrm{R}$ is independent of $\beta, \kappa, \gamma$ and $\lambda$.

\subsection{Multicomponent Stress-Strength Reliability Estimator $R_{s, m}$ Based on the MBXII-Power Distribution}

Suppose a machine has at least " $\mathrm{s}$ " components working out of "m" component. The strengths of all components of system are $\mathrm{X}_{1}, \mathrm{X}_{2}, \ldots . \mathrm{X}_{m}$ and stress $\mathrm{Y}$ is applied to the system. Both the strengths $\mathrm{X}_{1}, \mathrm{X}_{2}, \ldots . \mathrm{X}_{m}$ are i.i.d. and are independent of stress $\mathrm{Y}$. The cdf of $\mathrm{Y}$ is $\mathrm{G}$ and $\mathrm{F}$ is cdf of $\mathrm{X}$. The reliability of a machine is the probability that the machine functions properly. 
Let $X \sim M B X I I-\operatorname{Power}\left(\alpha_{1}, \beta, \gamma, \kappa, \lambda\right), Y \sim \operatorname{MBXII}-\operatorname{Power}\left(\alpha_{2}, \beta, \gamma, \kappa, \lambda\right)$ with common parameters $\beta, \kappa, \gamma$ and unknown shape parameters $\alpha_{1}$ and $\alpha_{2}$. The multicomponent stress- strength reliability for the MBXII-Power distribution is given by $R_{s, k}=P($ strengths $>$ stress $)=P\left[\right.$ at least "s" of $\left(\mathrm{X}_{1}, \mathrm{X}_{2}, \ldots . \mathrm{X}_{m}\right)$ exceed $\left.Y\right]$, $R_{s, m}=\sum_{l=s}^{m}\left(\begin{array}{l}k \\ \ell\end{array}\right) \int_{0}^{\infty}[1-F(y)]^{l}[F(y)]^{k-l} d G(y), \quad$ (Bhattacharyya and Johnson; 1974). $R_{s, m}=\sum_{l=s}^{m}\left(\begin{array}{l}m \\ l\end{array} \int_{0}^{\lambda}\left(\left[1+\gamma\left(\frac{x^{\kappa}}{\lambda^{\kappa}-x^{\kappa}}\right)^{\beta}\right]^{-\frac{\alpha_{1}}{\gamma}}\right)^{l}\left(1-\left[1+\gamma\left(\frac{x^{\kappa}}{\lambda^{\kappa}-x^{\kappa}}\right)^{\beta}\right]^{-\frac{\alpha_{1}}{\gamma}}\right)^{(m-l)} \frac{\alpha_{2} \beta \kappa \lambda^{\kappa} x^{\kappa \beta-1}}{\left(\lambda^{\kappa}-x^{\kappa}\right)^{\beta+1}}\left[1+\gamma\left(\frac{x^{\kappa}}{\lambda^{\kappa}-x^{\kappa}}\right)^{\beta}\right]^{-\frac{\alpha_{2}}{\gamma}-1} d x\right.$

Let $t=\left[1+\gamma\left(\frac{x^{\kappa}}{\lambda^{\kappa}-x^{\kappa}}\right)^{\beta}\right]^{-\frac{\alpha_{2}}{\gamma}}$, then we obtain

$$
R_{s, m}=\sum_{\ell=s}^{k}\left(\begin{array}{c}
m \\
\ell
\end{array}\right) \int_{0}^{1}\left(t^{v}\right)^{\ell}\left(1-t^{v}\right)^{(m-\ell)} d t
$$

Let $z=t^{v}$, then

$$
\begin{aligned}
& R_{s, m}=\frac{1}{v} \sum_{\ell=s}^{m}\left(\begin{array}{c}
m \\
\ell
\end{array}\right) \int_{0}^{1}(z)^{\ell+\frac{1}{v}-1}(1-z)^{(m-\ell)} d z, \\
& R_{s, m}=\frac{1}{v} \sum_{\ell=s}^{m}\left(\begin{array}{c}
m \\
\ell
\end{array}\right) B\left(\ell+\frac{1}{v}, m-\ell+1\right), \text { where } v=\frac{\alpha_{1}}{\alpha_{2}} .
\end{aligned}
$$

The probability $R_{s, m}$ in the (26) is called reliability in a multicomponent stress-strength model.

\section{CHARACTERIZATIONS}

In this section, the MBXII-Power distribution is characterized via: (i) conditional expectation; (ii) truncated moment; (iii) hazard function; (iv) Mills ratio; (v) certain functions of the random variable and (vi) conditional expectation of record values.

We present our characterizations in six subsections.

\subsection{Characterization Via Conditional Expectation}

The MBXII-Power distribution is characterized via conditional expectation..

Proposition 5.1.1: Let $X: \Omega \rightarrow(0, \lambda)$ be a continuous random variable with cdf $F(x)(0<F(x)<1$ for $x>0)$, then for $\alpha>\gamma, \mathrm{X}$ has cdf (4) if and only if 
Bhatti, F.A., Hamedani, G. G. Korkmaz, M. C. and Ahmad M,

$E\left[\left(\frac{X^{\kappa}}{\lambda^{\kappa}-X^{\kappa}}\right)^{\beta} \mid X>t\right]=\frac{1}{(\alpha-\gamma)}\left\{1+\alpha\left(\frac{t^{\kappa}}{\lambda^{\kappa}-t^{\kappa}}\right)^{\beta}\right\} \quad$ for $\alpha>\gamma$ and $0<\mathrm{t}<\lambda$

Proof. If X has cdf (4), then

$$
\begin{gathered}
E\left[\left(\frac{X^{\kappa}}{\lambda^{\kappa}-X^{\kappa}}\right)^{\beta} \mid X>t\right]=[1-F(t)]^{-1} \int_{t}^{\lambda}\left(\frac{x^{\kappa}}{\lambda^{\kappa}-x^{\kappa}}\right)^{\beta} f(x) d x, \\
=[1-F(t)]^{-1} \int_{t}^{\lambda}\left(\frac{x^{\kappa}}{\lambda^{\kappa}-x^{\kappa}}\right)^{\beta} \times \\
\frac{\alpha \beta \kappa \lambda^{\kappa} x^{\kappa \beta-1}}{\left(\lambda^{\kappa}-x^{\kappa}\right)^{\beta+1}}\left[1+\gamma\left(\frac{x^{\kappa}}{\lambda^{\kappa}-x^{\kappa}}\right)^{\beta}\right]^{-\frac{\alpha}{\gamma}-1} d x .
\end{gathered}
$$

Upon integration by parts and simplification, we arrive at

$$
E\left[\left(\frac{X^{\kappa}}{\lambda^{\kappa}-X^{\kappa}}\right)^{\beta} \mid X>t\right]=\frac{1}{(\alpha-\gamma)}\left\{1+\alpha\left(\frac{t^{\kappa}}{\lambda^{\kappa}-t^{\kappa}}\right)^{\beta}\right\} \quad \text { for } \alpha>\gamma \text { and } 0<\mathrm{t}<\lambda
$$

Conversely, if (27) holds, then

$$
\begin{aligned}
& \frac{1}{\bar{F}(t)} \int_{t}^{\infty}\left(\frac{x^{\kappa}}{\lambda^{\kappa}-x^{\kappa}}\right)^{\beta} f(x) d x=\frac{1}{(\alpha-\gamma)}\left\{1+\alpha\left(\frac{t^{\kappa}}{\lambda^{\kappa}-t^{\kappa}}\right)^{\beta}\right\}, \\
& \frac{1}{\bar{F}(t)} \int_{t}^{\infty}\left(\frac{x^{\kappa}}{\lambda^{\kappa}-x^{\kappa}}\right)^{\beta} f(x) d x=\left\{\left(\frac{t^{\kappa}}{\lambda^{\kappa}-t^{\kappa}}\right)^{\beta}+\frac{1}{(\alpha-\gamma)}\left[1+\gamma\left(\frac{t^{\kappa}}{\lambda^{\kappa}-t^{\kappa}}\right)^{\beta}\right]\right\} .
\end{aligned}
$$

Differentiating (28) with respect to t, we obtain

$$
-\left(\frac{t^{\kappa}}{\lambda^{\kappa}-t^{\kappa}}\right)^{\beta} f(t)=\bar{F}(t)\left[\frac{\beta \kappa \lambda^{\kappa} x^{\kappa \beta-1}}{\left(\lambda^{\kappa}-x^{\kappa}\right)^{\beta+1}}+\frac{1}{(\alpha-\gamma)} \frac{\beta \gamma \kappa \lambda^{\kappa} x^{\kappa \beta-1}}{\left(\lambda^{\kappa}-x^{\kappa}\right)^{\beta+1}}\right]-f(t)\left[\left(\frac{t^{\kappa}}{\lambda^{\kappa}-t^{\kappa}}\right)^{\beta}+\frac{1}{(\alpha-\gamma)}\left[1+\gamma\left(\frac{t^{\kappa}}{\lambda^{\kappa}-t^{\kappa}}\right)^{\beta}\right]\right]
$$

After simplification and integration we arrive at

$$
F(t)=1-\left[1+\gamma\left(\frac{t^{\kappa}}{\lambda^{\kappa}-t^{\kappa}}\right)^{\beta}\right]^{-\frac{\alpha}{\gamma}-1}, 0 \leq \mathrm{t} \leq \lambda
$$

\subsection{Characterizations via Truncated Moment of a Function of the Random Variable}

Here we characterize the MBXII-Power distribution via relationship between truncated moment of a function of $\mathrm{X}$ and another function. This characterization is stable in the sense of weak convergence (Glänzel; 1990). 
Proposition 5.2.1 Let $X: \Omega \rightarrow(0, \lambda)$ be a continuous random variable and let $g(x)=\left[1+\gamma\left(\frac{x^{\kappa}}{\lambda^{\kappa}-x^{\kappa}}\right)^{\beta}\right]^{-1}, 0<x<\lambda$. The pdf of $\mathrm{X}$ is pdf (5) if and only if the function $h(x)$, in Theorem $\mathrm{G}$ (Glänzel; 1990), has the form $h(x)=\frac{\alpha}{\alpha+\gamma}\left[1+\gamma\left(\frac{x^{\kappa}}{\lambda^{\kappa}-x^{\kappa}}\right)^{\beta}\right]^{-1}, 0<x<\lambda$.

Proof If $\mathrm{X}$ has pdf (5), then

$$
(1-F(x)) E(g(X) \mid X \geq x)=\frac{\alpha}{\alpha+\gamma}\left[1+\gamma\left(\frac{x^{\kappa}}{\lambda^{\kappa}-x^{\kappa}}\right)^{\beta}\right]^{-\left(\frac{\alpha}{\gamma}+1\right)}, 0<x<\lambda,
$$

or

$$
E(g(X) \mid X \geq x)=\frac{\alpha}{\alpha+\gamma}\left[1+\gamma\left(\frac{x^{\kappa}}{\lambda^{\kappa}-x^{\kappa}}\right)^{\beta}\right]^{-1}, 0<x<\lambda
$$

and

$$
h(x)-g(x)=-\frac{\gamma}{\alpha+\gamma}\left[1+\gamma\left(\frac{x^{\kappa}}{\lambda^{\kappa}-x^{\kappa}}\right)^{\beta}\right]^{-1}, 0<x<\lambda .
$$

Conversely, if $h(x)$ is given as above, then

$$
h^{\prime}(x)=-\frac{\alpha}{\alpha+\gamma} \gamma \frac{\beta \kappa \lambda^{\kappa} x^{\kappa \beta-1}}{\left(\lambda^{\kappa}-x^{\kappa}\right)^{\beta+1}}\left[1+\gamma\left(\frac{x^{\kappa}}{\lambda^{\kappa}-x^{\kappa}}\right)^{\beta}\right]^{-2}<0, \text { for } 0<x<\lambda,
$$

and

$$
s^{\prime}(x)=\frac{h^{\prime}(x)}{h(x)-g(x)}=\frac{\alpha \beta \kappa \lambda^{\kappa} x^{\kappa \beta-1}}{\left(\lambda^{\kappa}-x^{\kappa}\right)^{\beta+1}}\left[1+\gamma\left(\frac{x^{\kappa}}{\lambda^{\kappa}-x^{\kappa}}\right)^{\beta}\right]^{-1}, 0<x<\lambda,
$$

and hence

$$
s(x)=\ln \left[1+\gamma\left(\frac{x^{\kappa}}{\lambda^{\kappa}-x^{\kappa}}\right)^{\beta}\right]^{\frac{\alpha}{\gamma}}, 0<x<\lambda,
$$

and

$$
e^{-s(x)}=\left[1+\gamma\left(\frac{x^{\kappa}}{\lambda^{\kappa}-x^{\kappa}}\right)^{\beta}\right]^{-\frac{\alpha}{\gamma}}, 0<x<\lambda .
$$

In view of Theorem $\mathrm{G}, \mathrm{X}$ has density (4).

Corollary 5.2.1: Let $X: \Omega \rightarrow(0, \lambda)$ be a continuous random variable. The pdf of $X$ is (5) if and only if there exist functions $h(x)$ and $g(x)$ defined in Theorem $\mathrm{G}$ satisfying the differential equation 


$$
s^{\prime}(x)=\frac{\alpha \beta \kappa \lambda^{\kappa} x^{\kappa \beta-1}}{\left(\lambda^{\kappa}-x^{\kappa}\right)^{\beta+1}}\left[1+\gamma\left(\frac{x^{\kappa}}{\lambda^{\kappa}-x^{\kappa}}\right)^{\beta}\right]^{-1}, 0<x<\lambda .
$$

Remark 5.2.1: The general solution of the differential equation in Corollary 5.2.1 is

$$
h(x)=\left[1+\gamma\left(\frac{x^{\kappa}}{\lambda^{\kappa}-x^{\kappa}}\right)^{\beta}\right]^{\frac{\alpha}{\gamma}}\left\{-\int \frac{\alpha \beta \kappa \lambda^{\kappa} x^{\kappa \beta-1}}{\left(\lambda^{\kappa}-x^{\kappa}\right)^{\beta+1}}\left[1+\gamma\left(\frac{x^{\kappa}}{\lambda^{\kappa}-x^{\kappa}}\right)^{\beta}\right]^{-1} g(x) d x+D\right\},
$$

where $\mathrm{D}$ is a constant.

\subsection{Characterization via Hazard Function}

In this sub-section, the MBXII-Power distribution is characterized via hazard function.

Definition 5.3.1: Let $\mathrm{X}$ be a continuous random variable with pdf $f(x)$. The hazard function $h_{F}(x)$ of a twice differentiable distribution function satisfies the differential equation

$$
\frac{d}{d x}[\ln f(x)]=\frac{h_{F}^{\prime}(x)}{h_{F}(x)}-h_{F}(x)
$$

Proposition 5.3.1 Let $X: \Omega \rightarrow(0, \lambda)$ be continuous random variable .The pdf of $X$ is (5) if and only if its hazard function, $h_{F}(x)$, satisfies the first order differential equation

$$
x h_{F}^{\prime}(x)+h_{F}(x)=\alpha \beta \kappa \lambda^{\kappa} \frac{\kappa x^{\kappa \beta-1}\left(\lambda^{\kappa}-x^{\kappa}\right)^{-\beta-2}}{\left[1+\gamma x^{\kappa \beta}\left(\lambda^{\kappa}-x^{\kappa}\right)^{-\beta}\right]}\left\{\beta \lambda^{\kappa}+x^{\kappa}+\frac{\beta \lambda^{\kappa} x^{\kappa \beta}\left(\lambda^{\kappa}-x^{\kappa}\right)^{-\beta}}{\left[1+\gamma x^{\kappa \beta}\left(\lambda^{\kappa}-x^{\kappa}\right)^{-\beta}\right]}\right\} .
$$

Proof. If $\mathrm{X}$ has pdf (5), then the above differential equation holds. Now if the differential equation holds, then

or

$$
\begin{aligned}
& \frac{d}{d x}\left\{x h_{f}(x)\right\}=\alpha \beta \kappa \lambda^{\kappa} \frac{d}{d x}\left\{\frac{x^{\kappa \beta}}{\left(\lambda^{\kappa}-x^{\kappa}\right)^{\beta+1}}\left[1+\gamma\left(\frac{x^{\kappa}}{\lambda^{\kappa}-x^{\kappa}}\right)^{\beta}\right]^{-1}\right\}, \\
& h(x)=\frac{\alpha \beta \kappa \lambda^{\kappa} x^{\kappa \beta-1}}{\left(\lambda^{\kappa}-x^{\kappa}\right)^{\beta+1}}\left[1+\gamma\left(\frac{x^{\kappa}}{\lambda^{\kappa}-x^{\kappa}}\right)^{\beta}\right]^{-1}, 0<x<\lambda,
\end{aligned}
$$

which is the hazard function of the MBXII-Power distribution.

\subsection{Characterization via Mills Ratio}

In this sub-section, the MBXII-Power distribution is characterized via Mills ratio. 
Definition 5.4.1: Let $X: \Omega \rightarrow(0, \lambda)$ be a continuous random variable with cdf $F(x)$ and pdf $f(x)$.The Mills ratio, $m(x)$, of a twice differentiable function, $\mathrm{F}$, satisfies the first order differential equation

$$
\frac{d}{d x}[\ln f(x)]=-\left[\frac{1}{m(x)}+\frac{m^{\prime}(x)}{m(x)}\right]
$$

Proposition 5.4.1: Let $X: \Omega \rightarrow(0, \lambda)$ be continuous random variable .The pdf of $X$ is $(5)$ if and only if the Mills ratio satisfies the first order differential equation

$$
m_{F}^{\prime}(x)+m_{F}(x)(\kappa \beta-1) x^{-1}=\left\{\frac{\gamma}{\alpha}+\frac{(\beta+1)}{\alpha \beta \lambda^{\kappa}}\left[x^{\kappa-\kappa \beta}\left(\lambda^{\kappa}-x^{\kappa}\right)^{\beta}+\gamma x^{\kappa}\right]\right\} .
$$

Proof If $\mathrm{X}$ has pdf (5), then the above differential equation surely holds. Now if the differential equation holds, then

or

$$
\begin{aligned}
& \frac{d}{d x}\left[m_{F}(x) \alpha \beta \kappa \lambda^{\kappa} x^{\kappa \beta-1}\right]=\frac{d}{d x}\left[\left(\lambda^{\kappa}-x^{\kappa}\right)^{\beta+1}\left(1+\gamma\left(\frac{x^{\kappa}}{\lambda^{\kappa}-x^{\kappa}}\right)^{\beta}\right)\right], \\
& m(x)=\frac{\left[1+\gamma\left(\frac{x^{\kappa}}{\lambda^{\kappa}-x^{\kappa}}\right)^{\beta}\right]}{\alpha \beta \kappa \lambda^{\kappa} x^{\kappa \beta-1}\left(\lambda^{\kappa}-x^{\kappa}\right)^{-\beta-1}},
\end{aligned}
$$

which is Mills ratio of the MBXII-Power distribution.

\subsection{Characterization via Certain Function of the Random Variable}

The MBXII-Power distribution is characterized through certain function of the continuous random variable X. Hamedani (2013) used this technique for characterization.

Proposition 5.5.1. Let $\mathrm{X}: \Omega \rightarrow(0, \lambda)$ be continuous random variable with cdf $F(x)$ and pdf $f(x)$. Let $\psi(x)$ and $\varphi(x)$ be differentiable functions on $(0, \lambda)$ such that $\int_{0}^{\lambda} \frac{\psi^{\prime}(x)}{\psi(x)-\varphi(x)} d x=\lambda$. Then $E(\varphi(X) \mid X>x)=\psi(x), 0<x<\lambda$, implies

$$
F(x)=1-\exp \left[-\int_{0}^{x} \frac{\psi^{\prime}(t)}{\psi(t)-\varphi(t)} d t\right], 0 \leq x \leq \lambda
$$

Proof. We have $\int_{x}^{\lambda} \varphi(u) f(u) d u=(1-F(x)) \psi(x)$.

After differentiation the above equation with respect to $\mathrm{x}$, and then reorganizing the terms, we obtain 


$$
\frac{f(x)}{1-F(x)}=\frac{\psi^{\prime}(x)}{\psi(x)-\varphi(x)}, 0<x<\lambda \text {. }
$$

Integrating the last equation from 0 to $\mathrm{x}$, we have

$$
F(x)=1-\exp \left[-\int_{0}^{x} \frac{\psi^{\prime}(t)}{\psi(t)-\varphi(t)} d t\right], 0 \leq x \leq \lambda .
$$

Remark 5.5.1. Taking $\varphi(x)=\left[1+\gamma\left(\frac{x^{\kappa}}{\lambda^{\kappa}-x^{\kappa}}\right)^{\beta}\right]^{\frac{\alpha}{2 \gamma}}$ and $\psi(x)=2 \varphi(x)$, Proposition 5.5.1 provides a characterization of (4). Clearly there are other choices of these functions.

\subsection{Characterization via Conditional Expectation of the Record Values}

Nagaraja (1988), Arnold et al. (1998), Khan and Alzaid (2004), Khan et al. (2010) and Athar et al. (2014) characterized distributions via conditional expectation of the record values.

Proposition 5.6.1: Let $\mathrm{X}: \Omega \rightarrow(0, \lambda)$ be a continuous random variable with cdf $F(x)$ and pdf $f(x)$. Let $X_{U(r)}$ be the $r t h$ record value of a random sample $X_{1}, X_{2}, \ldots \mathrm{X}_{n}$. Then for two successive values $X_{U(r)}$ and $X_{U(\mathrm{~s})}, 1 \leq r<s \leq n$, $E\left[\left(\mathrm{~h}\left(X_{U(s)}-X_{U(r)}\right)\right)^{q} \mid X_{U(r)}=x\right]=a^{*} \sum_{j=0}^{q}\left(\begin{array}{c}q \\ j\end{array}\right)(\mathrm{h}(x))^{q-j}(b / a)^{j}$ holds, if and only if $F(x)=1-[a+b h(x)]^{c}, a \neq 0$, where $a^{*}=\sum_{i=0}^{q}\left(\begin{array}{l}q \\ i\end{array}\right)(-1)^{i+q}\left(\frac{c}{c+i}\right)^{s-r}$ and $h(x)$ is a differentiable function of $x$.

Remark 5.6.1: Taking $a=1, b=\gamma, h(x)=\left(\frac{x^{\kappa}}{\lambda^{\kappa}-x^{\kappa}}\right)^{\beta}, c=-\frac{\alpha}{\gamma}$, proposition 5.6.1 provides a characterization of the MBXII-Power distribution.

\section{MAXIMUM LIKELIHOOD ESTIMATION}

In this section, parameters estimates are derived using maximum likelihood method. The $\log$-likelihood function for the vector of parameters $\Phi=(\alpha, \beta, \gamma, \kappa, \lambda)$ of the MBXIIPower distribution is

$$
\begin{aligned}
& \operatorname{lnL}(\Phi)=n \ln (\alpha)+n \ln (\beta)+n \ln (\kappa)+n \kappa \ln (\lambda)+(\kappa \beta-1) \sum_{i=1}^{n} \ln x_{i}- \\
& (\beta+1) \sum_{i=1}^{n} \ln \left(\lambda^{\kappa}-x_{i}{ }^{\kappa}\right)-\left(\frac{\alpha}{\gamma}+1\right) \sum_{i=1}^{n} \ln \left[1+\gamma\left(\frac{x_{i}{ }^{\kappa}}{\lambda^{\kappa}-x_{i}{ }^{\kappa}}\right)^{\beta}\right]
\end{aligned}
$$


Where $\lambda$ is assumed to known because of its maximum likelihood is equal to maximum order statistics. In order to compute the estimates of the parameters $\alpha, \beta, \gamma, \kappa$ of the MBXII-Power distribution, the following nonlinear equations must be solved simultaneously:

$$
\begin{aligned}
& \frac{\partial}{\partial \alpha} \ell(\Phi)=\frac{n}{\alpha}-\frac{1}{\gamma} \sum_{i=1}^{n} \ln \left[1+\gamma\left(\frac{x_{i}{ }^{\kappa}}{\lambda^{\kappa}-x_{i}{ }^{\kappa}}\right)^{\beta}\right]=0 \\
& \frac{\partial}{\partial \beta} \ell(\Phi)=\frac{n}{\beta}+\kappa \sum_{i=1}^{n} \ln x_{i}-\sum_{i=1}^{n} \ln \left(\lambda^{\kappa}-x_{i}{ }^{\kappa}\right)-(\alpha+\gamma) \sum_{i=1}^{n}\left[\left(\frac{x_{i}{ }^{\kappa}}{\lambda^{\kappa}-x_{i}{ }^{\kappa}}\right)^{-\beta}+\gamma\right]^{-1}\left[\ln \left(\frac{x_{i}^{\kappa}}{\lambda^{\kappa}-x_{i}{ }^{\kappa}}\right)\right],(3
\end{aligned}
$$

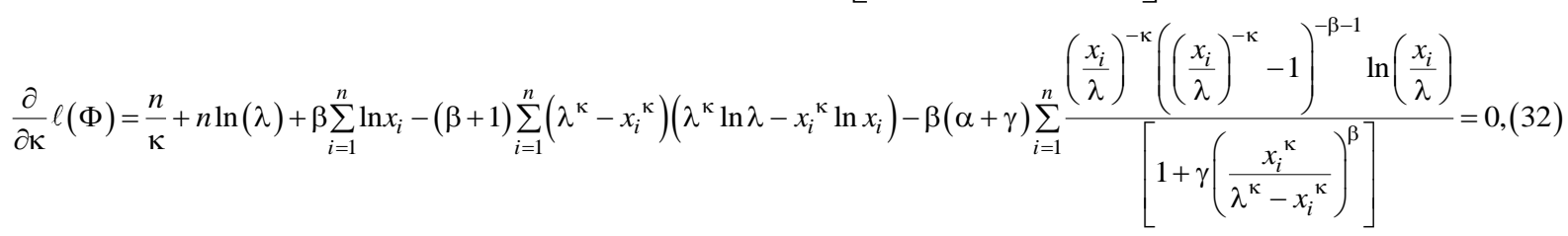

$$
\begin{aligned}
& \frac{\partial}{\partial \gamma} \ell(\Phi)=\frac{\alpha}{\gamma^{2}} \sum_{i=1}^{n} \ln \left[1+\gamma\left(\frac{x_{i}{ }^{\kappa}}{\lambda^{\kappa}-x_{i}{ }^{\kappa}}\right)^{\beta}\right]-\left(\frac{\alpha}{\gamma}+1\right) \sum_{i=1}^{n}\left[\left(\frac{x_{i}{ }^{\kappa}}{\lambda^{\kappa}-x_{i}{ }^{\kappa}}\right)^{-\beta}+\gamma\right]^{-1} .
\end{aligned}
$$

The above equations 30-33 can be solved either directly or using the R (optim and maxLik functions), SAS (PROC NLMIXED) and Ox program (sub-routine Max BFGS), or employing non-linear optimization methods such as the quasi-Newton algorithm.

\section{SIMULATION STUDY}

In this Section, we perform simulation study based on graphical results by using the MBXII-Power distribution to see the performance of MLEs corresponding to this distribution. The random numbers generation is obtained by the its quantile function. All results related to MLEs were obtained using optim-CG routine in the R programme.

We generate $\mathrm{N}=1000$ samples of size $\mathrm{n}=20,25, \ldots, 1000$ from the MBXII-Power distribution with true parameters values $\alpha=10, \beta=4, \gamma=5, \lambda=0.5$ and $\kappa=8$. In this simulation study, we empirically calculate the mean, standard deviations (sd), bias and mean square error (MSE) of the MLEs. The bias and MSE are calculated by (for

$$
h=\alpha, \beta, \gamma, \lambda, \kappa) \quad \operatorname{Bias}_{h}=\frac{1}{1000} \sum_{i=1}^{1000}\left(\widehat{h}_{i}-h\right) \quad \text { and } \quad M S E_{h}=\frac{1}{1000} \sum_{i=1}^{1000}\left(\hat{h}_{i}-h\right)^{2} \text {, }
$$

respectively. We give results of this simulation study in Figure 4. From Figure 4, we observe that when the sample size increases, the empirical means approach the true parameter value whereas all biases, sds and MSEs approach to 0 in all cases. 

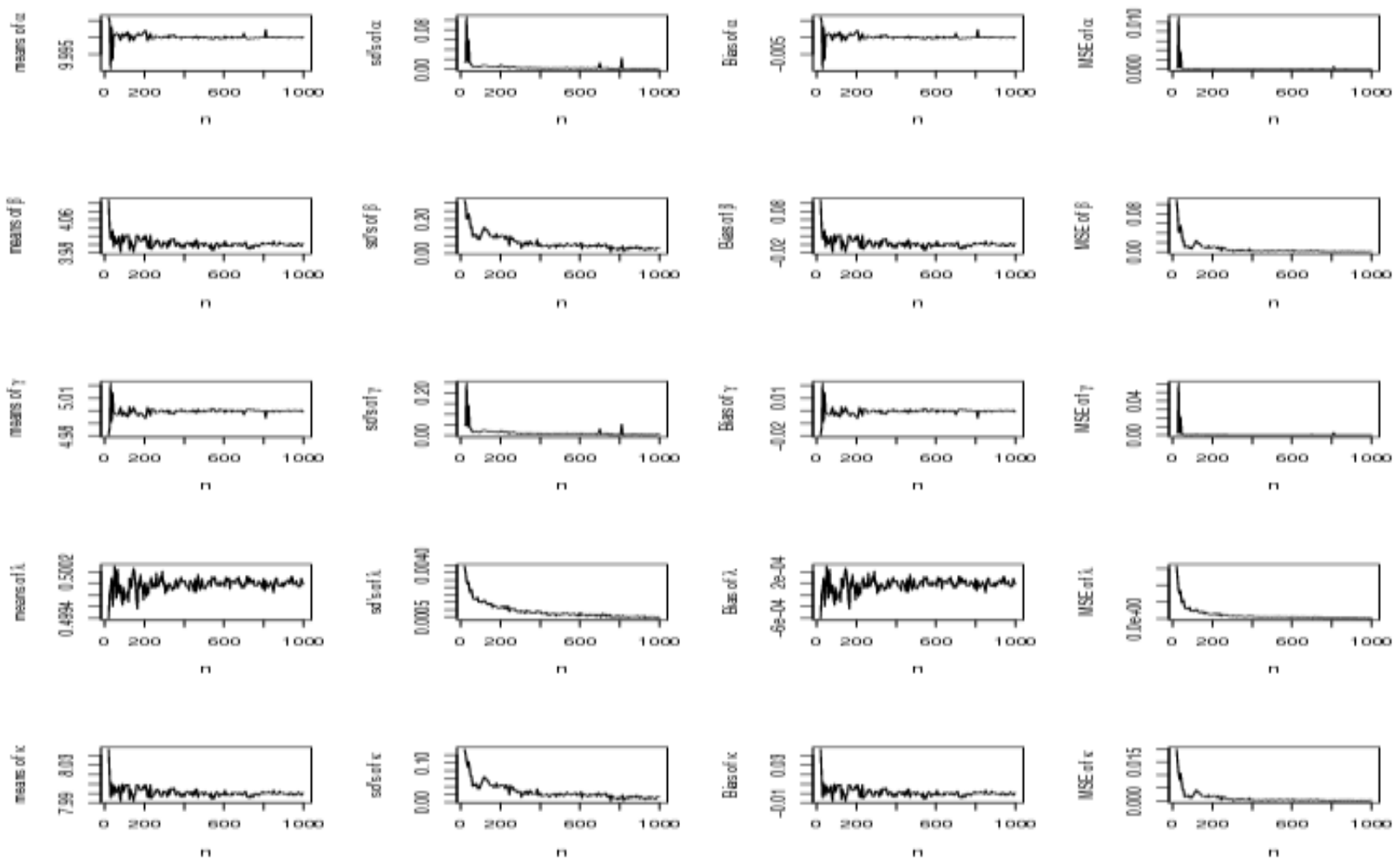

Fig. 4: Simulation results of the MBXII-Power distribution

\section{APPLICATIONS}

In this section, the MBXII-Power distribution is compared with BXII-Power, LomaxPower, LL-Power, Weibull Power, Power, modified Burr XII (MBXII), modified Burr III (MBIII), Weibull and Inverse Weibull (IW) distributions. Different goodness of fit measures such as Cramer-von Mises (W), Anderson Darling (A), Kolmogorov- Smirnov (K-S) statistics with p-values, Akaike Information Criterion (AIC), consistent Akaike Information Criterion (CAIC), Bayesian Information Criterion (BIC), Hannan-Quinn Information Criterion (HQIC) and likelihood ratio statistics are computed using Rpackage for real data sets: survival times of pigs, survival times of patients and quarterly earnings. The better fit corresponds to smaller W, A, K-S, AIC, CAIC, BIC, HQIC and $-\ell$ value. The maximum likelihood estimates (MLEs) of unknown parameters and values of goodness of fit measures are computed for the MBXII-Power distribution and its sub and competing models. The MLEs, their standard errors (in parentheses) and goodness-of-fit statistics like W, A, K-S (P-values) are given in tables 3, 5 and 7. Tables 4, 6 and 8 display goodness-of-fit values. 
8.1 Application I: Survival Times for Guinea Pigs: The survival times for guinea pigs injected with different doses of tubercle bacilli are 12, 15, 22, 24, 24, 32, 32, 33, 34, 38, $38,43,44,48,52,53,54,54,55,56,57,58,58,59,60,60,60,60,61,62,63,65,65,67$, $68,70,70,72,73,75,76,76,81,83,84,85,87,91,95,96,98,99,109,110,121,127$, $129,131,143,146,146,175,175,211,233,258,258,263,297,341,341,376$.

Table 3: MLEs, their standard errors (in parentheses) and Goodness-of-fit statistics for data set I

\begin{tabular}{|l|l|l|l|l|l|l|l|l|}
\hline Model & $\alpha$ & $\beta$ & $\gamma$ & $\kappa$ & $\lambda$ & W & A & $\begin{array}{l}\text { K-S } \\
\text { p-value }\end{array}$ \\
\hline $\begin{array}{l}\text { MBXII- } \\
\text { Power }\end{array}$ & $\begin{array}{l}104.663 \\
(105.162)\end{array}$ & $\begin{array}{l}0.0173 \\
(0.0152)\end{array}$ & $\begin{array}{l}130.378 \\
(176.708)\end{array}$ & $\begin{array}{l}162.138 \\
(148.58)\end{array}$ & 376 & 0.0853 & 0.4921 & $\begin{array}{l}\mathbf{0 . 0 8 3 2} \\
(\mathbf{0 . 7 0 9 3})\end{array}$ \\
\hline $\begin{array}{l}\text { BXII- } \\
\text { Power }\end{array}$ & $\begin{array}{l}7.7504 \\
(1.2988)\end{array}$ & $\begin{array}{l}0.2020 \\
(0.1298)\end{array}$ & & $7.6652(5.070)$ & 376 & 0.3446 & 1.8884 & $\begin{array}{l}0.1293 \\
(0.1861)\end{array}$ \\
\hline $\begin{array}{l}\text { L- } \\
\text { Power }\end{array}$ & $\begin{array}{l}3.3493 \\
(0.6148)\end{array}$ & & $1.1719(0.130)$ & 376 & 0.6358 & 3.4809 & $\begin{array}{l}0.1877 \\
(0.0134)\end{array}$ \\
\hline $\begin{array}{l}\text { LL- } \\
\text { Power }\end{array}$ & & $\begin{array}{l}2.4258 \\
(0.2596)\end{array}$ & & $0.4488(0.027)$ & 376 & 0.4458 & 2.4547 & $\begin{array}{l}0.1208 \\
(0.2514)\end{array}$ \\
\hline Power & & & $0.6220(0.074)$ & 376 & 0.5637 & 3.0805 & $\begin{array}{l}0.304 \\
(0.0000)\end{array}$ \\
\hline $\begin{array}{l}\text { W- } \\
\text { Power }\end{array}$ & $\begin{array}{l}5.9814 \\
(1.0229)\end{array}$ & $\begin{array}{l}0.1489 \\
(0.1058)\end{array}$ & & $9.5791(6.988)$ & 376 & 0.4079 & 2.2437 & $\begin{array}{l}0.1424 \\
(0.1123)\end{array}$ \\
\hline MBXII & $\begin{array}{l}0.0117 \\
(0.0195)\end{array}$ & $\begin{array}{l}100.528 \\
(779.34)\end{array}$ & $\begin{array}{c}5.099 \\
(46.522)\end{array}$ & & & 0.1347 & 0.7571 & $\begin{array}{l}0.4827 \\
(0.0000)\end{array}$ \\
\hline MBIII & $\begin{array}{l}19759.19 \\
(9642.85)\end{array}$ & $\begin{array}{l}2.31708 \\
(0.1168)\end{array}$ & $\begin{array}{l}14490.76 \\
(8808.61)\end{array}$ & & & 0.096 & 0.5504 & $\begin{array}{l}0.0874 \\
(0.6406)\end{array}$ \\
\hline Weibull & $\begin{array}{l}0.0032 \\
(0.0007)\end{array}$ & $\begin{array}{l}1.2368 \\
(0.0487)\end{array}$ & & & & 0.3974 & 2.1842 & $\begin{array}{l}0.1532 \\
(0.0683)\end{array}$ \\
\hline $\begin{array}{l}\text { Inverse } \\
\text { Weibull }\end{array}$ & $\begin{array}{l}283.8278 \\
(125.6736\end{array}$ & $\begin{array}{l}1.4147 \\
(0.1173)\end{array}$ & & & & 0.2148 & 1.2833 & $\begin{array}{l}0.152 \\
(0.0719)\end{array}$ \\
\hline
\end{tabular}

Table 4: Goodness-of-fit statistics for data set I

\begin{tabular}{|l|r|r|r|r|r|}
\hline Model & \multicolumn{1}{|c|}{ AIC } & \multicolumn{1}{l|}{ CAIC } & \multicolumn{1}{l|}{ BIC } & \multicolumn{1}{l|}{ HQIC } & \multicolumn{1}{l|}{$\ell$} \\
\hline MBXII- Power & 769.08 & 769.69 & 778.13 & 772.68 & 380.54 \\
\hline BXII- Power & 776.61 & 776.97 & 783.40 & 779.31 & 385.30 \\
\hline L- Power & 790.68 & 790.85 & 795.20 & 792.47 & 393.34 \\
\hline LL- Power & 779.88 & 780.06 & 784.41 & 781.68 & 387.94 \\
\hline Power & 825.14 & 825.19 & 827.40 & 826.04 & 411.57 \\
\hline W-Power & 780.13 & 780.49 & 786.92 & 782.83 & 387.06 \\
\hline MBXII & 987.64 & 987.99 & 994.47 & 990.35 & 490.82 \\
\hline MBIII & 785.35 & 785.70 & 792.18 & 788.07 & 389.67 \\
\hline Weibull & 800.20 & 800.38 & 804.76 & 802.01 & 398.10 \\
\hline Inverse Weibull & 795.30 & 795.47 & 799.85 & 797.11 & 395.65 \\
\hline
\end{tabular}

The MBXII-Power distribution is best fitted model than the other sub-models because the 
Bhatti, F.A., Hamedani, G. G. Korkmaz, M. C. and Ahmad M,

values of all criteria of goodness of fit are significantly smaller for the MBXII-Power distribution.
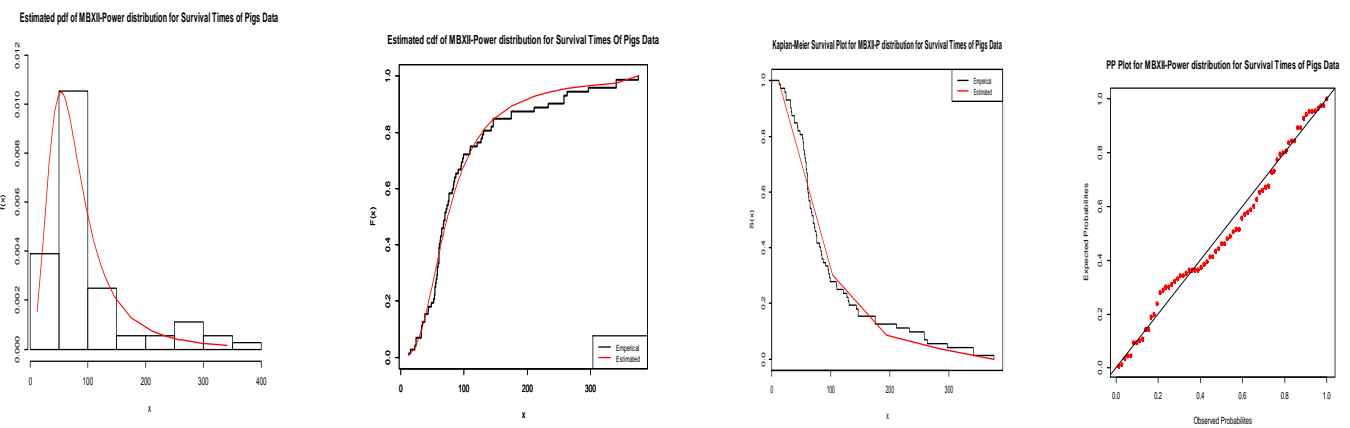

Fig. 5: Fitted pdf, cdf, survival and pp plots of the MBXII-Power distribution for Survival Times of Pigs data

We can observe that the MBXII-Power distribution is close to empirical data (Fig. 5).

8.2 Application II: Survival Times of Patients: The data are collected (Feigl and Zelen; 1965) about the survival times (weeks) of 33 patients suffering from acute Myelogeneous Leukaemia (Mead et al.; 2017). The data are: 65, 156, 100, 134, 16, 108, 121, 4, 39, 143, 56, 26, 22, 1, 1, 5, 65, 56, 65, 17, 7, 16, 22, 3, 4, 2, 3, 8, 4, 3, 30, 4, 43.

Table 5: MLEs, their standard errors (in parentheses) and Goodness-of-fit statistics for data set II

\begin{tabular}{|c|c|c|c|c|c|c|c|c|}
\hline Model & $\alpha$ & $\beta$ & $\gamma$ & $\kappa$ & $\lambda$ & W & A & $\begin{array}{l}\mathrm{K}-\mathrm{S} \\
\mathrm{p} \text {-value }\end{array}$ \\
\hline $\begin{array}{l}\text { MBXII- } \\
\text { Power }\end{array}$ & $\begin{array}{l}20.189 \\
(6.022)\end{array}$ & $\begin{array}{l}8.566 \\
(2.896)\end{array}$ & $\begin{array}{l}236.078 \\
(3.357)\end{array}$ & $\begin{array}{l}0.231 \\
(0.040)\end{array}$ & 156 & 0.0534 & 0.3181 & $\begin{array}{l}0.1145 \\
(0.7949)\end{array}$ \\
\hline $\begin{array}{l}\text { BXII- } \\
\text { Power }\end{array}$ & $\begin{array}{l}3.537 \\
(1.078) \\
\end{array}$ & $\begin{array}{l}0.406 \\
(0.198) \\
\end{array}$ & ------- & $\begin{array}{l}1.880 \\
(1.096)\end{array}$ & 156 & 0.0702 & 0.4776 & $\begin{array}{l}0.1244 \\
(0.7049)\end{array}$ \\
\hline $\begin{array}{l}\text { L- } \\
\text { Power }\end{array}$ & $\begin{array}{l}1.524 \\
(0.370)\end{array}$ & $\begin{array}{l}------- \\
-- \\
\end{array}$ & ------- & $\begin{array}{l}0.560 \\
(0.113)\end{array}$ & 156 & 0.1175 & 0.7577 & $\begin{array}{l}0.1325 \\
(0.6282)\end{array}$ \\
\hline $\begin{array}{l}\text { LL- } \\
\text { Power }\end{array}$ & --------- & $\begin{array}{l}1.329(0 . \\
214)\end{array}$ & ------- & $\begin{array}{l}0.374 \\
(0.061)\end{array}$ & 156 & 0.1008 & 0.6935 & $\begin{array}{l}0.1265 \\
(0.6850)\end{array}$ \\
\hline Power & & & ------- & $\begin{array}{l}0.437 \\
(0.077)\end{array}$ & 156 & 0.104 & 0.7115 & $\begin{array}{l}0.1615 \\
(0.3741)\end{array}$ \\
\hline $\begin{array}{l}\text { W- } \\
\text { Power }\end{array}$ & $\begin{array}{l}2.7612 \\
(0.771)\end{array}$ & $\begin{array}{l}0.282 \\
(0.128)\end{array}$ & ------- & $\begin{array}{l}2.494 \\
(1.352)\end{array}$ & 156 & 0.0809 & 0.5488 & $\begin{array}{l}0.1254 \\
(0.6951)\end{array}$ \\
\hline MBXII & $\begin{array}{l}0.0628 \\
(0.0298)\end{array}$ & $\begin{array}{l}0.776 \\
(0.236)\end{array}$ & $\begin{array}{l}0.0000000001 \\
(0.057)\end{array}$ & & ----- & 0.0948 & 0.6508 & $\begin{array}{l}0.1366 \\
(0.5692) \\
\end{array}$ \\
\hline MBIII & $\begin{array}{l}144203.0 \\
(38.083)\end{array}$ & $\begin{array}{l}2.950 \\
(0.131)\end{array}$ & $\begin{array}{l}778070.4 \\
(3294.290) \\
\end{array}$ & $\begin{array}{l}------- \\
-\end{array}$ & ------ & 0.1111 & 0.7691 & $\begin{array}{l}0.1416 \\
(0.5222)\end{array}$ \\
\hline Weibull & $\begin{array}{l}0.0628 \\
(0.030)\end{array}$ & $\begin{array}{l}0.776 \\
(0.107)\end{array}$ & ---------- & $\begin{array}{l}------- \\
--\end{array}$ & ------ & 0.0948 & 0.6508 & $\begin{array}{l}0.1366 \\
(0.5692) \\
\end{array}$ \\
\hline $\begin{array}{l}\text { Inverse } \\
\text { Weibull }\end{array}$ & $\begin{array}{l}4.188 \\
(0.903)\end{array}$ & $\begin{array}{l}0.694 \\
(0.0915)\end{array}$ & ---------- & --------- & ----- & 0.1601 & 0.9759 & $\begin{array}{l}0.149 \\
(0.4561)\end{array}$ \\
\hline
\end{tabular}


Table 6: Goodness-of-fit statistics for data set II

\begin{tabular}{|l|r|r|r|r|r|}
\hline Model & \multicolumn{1}{|l|}{ AIC } & CAIC & \multicolumn{1}{l|}{ BIC } & \multicolumn{1}{l|}{ HQIC } & $-\ell$ \\
\hline MBXII- Power & 291.14 & 292.62 & 297.00 & 293.08 & 141.57 \\
\hline BXII- Power & 293.80 & 294.65 & 298.19 & 295.25 & 143.90 \\
\hline L- Power & 294.86 & 295.27 & 297.79 & 295.83 & 145.43 \\
\hline LL- Power & 295.00 & 295.41 & 297.93 & 295.97 & 145.50 \\
\hline Power & 295.68 & 295.81 & 297.14 & 296.16 & 146.84 \\
\hline W-Power & 294.11 & 294.97 & 298.51 & 295.57 & 144.05 \\
\hline MBXII & 313.17 & 314.00 & 317.66 & 314.68 & 153.59 \\
\hline MBIII & 315.50 & 316.33 & 319.99 & 317.01 & 154.75 \\
\hline Weibull & 311.17 & 311.57 & 314.17 & 312.18 & 153.59 \\
\hline Inverse Weibull & 316.00 & 316.40 & 318.99 & 317.00 & 156.00 \\
\hline
\end{tabular}

The MBXII-Power distribution is best fitted model than the other sub-models because the values of all criteria of goodness of fit are significantly smaller for the MBXII-Power distribution.
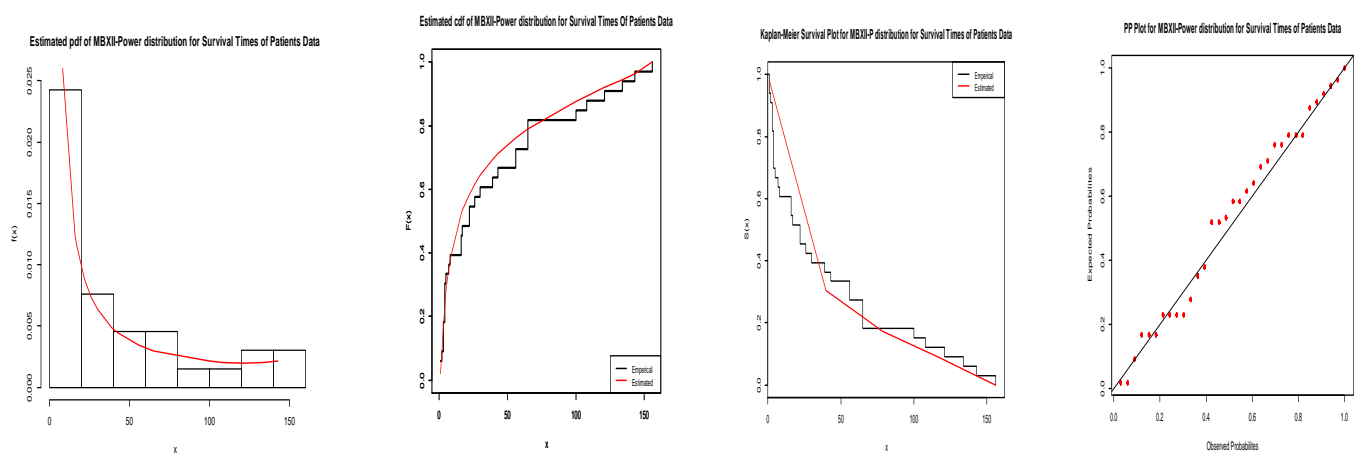

Fig. 6: Fitted pdf, cdf, survival and pp plots of the MBXII-Power distribution for Survival Times of Patients

We can observe that the MBXII-Power distribution is close to empirical data (Fig.6).

8.3 Application III: Quarterly Earnings: The second data set are the quarterly earnings per Johnson and Johnson Share (1960 to 1980) Source R package. The data are: $0.71,0.63,0.85,0.44,0.61,0.69,0.92,0.55,0.72,0.77,0.92,0.60,0.83,0.80,1.00,0.77$, $0.92,1.00,1.24,1.00,1.16,1.30,1.45,1.25,1.26,1.38,1.86,1.56,1.53,1.59,1.83,1.86$, $1.53,2.07,2.34,2.25,2.16,2.43,2.70,2.25,2.79,3.42,3.69,3.60,3.60,4.32,4.32,4.05$, $4.86,5.04,5.04,4.41,5.58,5.85,6.5,5.31,6.03,6.39,6.93,5.85,6.93,7.74,7.83,6.12$, $7.74,8.91,8.28,6.84,9.54,10.26,9.54,8.73,11.88,12.06,12.15,8.91,14.04,12.96$, 14.85 . 
Bhatti, F.A., Hamedani, G. G. Korkmaz, M. C. and Ahmad M,

Table 7: MLEs, their standard errors (in parentheses) and Goodness-of-fit statistics for data set III

\begin{tabular}{|c|c|c|c|c|c|c|c|c|}
\hline Model & $\alpha$ & $\beta$ & $\gamma$ & $\kappa$ & $\lambda$ & W & A & $\begin{array}{l}\text { K-S } \\
\text { p-value }\end{array}$ \\
\hline $\begin{array}{l}\text { MBXII- } \\
\text { Power }\end{array}$ & $\begin{array}{l}0.023 \\
(0.042)\end{array}$ & $\begin{array}{l}16.251 \\
(6.653)\end{array}$ & $\begin{array}{c}0.391 \\
(0.7397)\end{array}$ & $\begin{array}{r}0.2066 \\
(0.0185)\end{array}$ & 14.85 & 0.0733 & 0.4117 & $\begin{array}{l}0.083 \\
(0.656)\end{array}$ \\
\hline $\begin{array}{l}\text { BXII- } \\
\text { Power }\end{array}$ & $\begin{array}{l}4.575 \\
(0.883) \\
\end{array}$ & $\begin{array}{l}0.404 \\
(0.153) \\
\end{array}$ & & $\begin{array}{l}2.886 \\
(1.251) \\
\end{array}$ & 14.85 & 0.2108 & 1.2969 & $\begin{array}{l}0.1067 \\
(0.337) \\
\end{array}$ \\
\hline $\begin{array}{l}\text { L- } \\
\text { Power }\end{array}$ & $\begin{array}{l}2.161 \\
(0.358) \\
\end{array}$ & & & $\begin{array}{l}0.903 \\
(0.1079)\end{array}$ & 14.85 & 0.2513 & 1.6211 & $\begin{array}{l}0.1258 \\
(0.169) \\
\end{array}$ \\
\hline $\begin{array}{l}\text { LL- } \\
\text { Power }\end{array}$ & & $\begin{array}{l}1.643 \\
(0.163)\end{array}$ & & $\begin{array}{l}0.463 \\
(0.0395)\end{array}$ & 14.85 & 0.2546 & 1.6031 & $\begin{array}{l}0.1118 \\
(0.284)\end{array}$ \\
\hline Power & & & & $\begin{array}{l}0.5848 \\
(0.0662)\end{array}$ & 14.85 & 0.2359 & 1.5215 & $\begin{array}{l}0.1673 \\
(0.025) \\
\end{array}$ \\
\hline $\begin{array}{l}\text { W- } \\
\text { Power }\end{array}$ & $\begin{array}{l}3.692 \\
(0.662) \\
\end{array}$ & $\begin{array}{l}0.267 \\
(0.109)\end{array}$ & & $\begin{array}{l}4.0888 \\
(1.8684)\end{array}$ & 14.85 & 0.2196 & 1.3586 & $\begin{array}{l}0.1101 \\
(0.301) \\
\end{array}$ \\
\hline MBXII & $\begin{array}{l}3.692 \\
(0.040)\end{array}$ & $\begin{array}{l}0.267 \\
(0.222) \\
\end{array}$ & $\begin{array}{l}4.0888 \\
(0.069) \\
\end{array}$ & & & 0.2424 & 1.4901 & $\begin{array}{l}0.1158 \\
(0.240) \\
\end{array}$ \\
\hline MBIII & $\begin{array}{l}1.797 \\
(0.373)\end{array}$ & $\begin{array}{l}1.126 \\
(80.129)\end{array}$ & $\begin{array}{l}0.0000000001 \\
(0.294)\end{array}$ & & & 0.2745 & 1.6926 & $\begin{array}{l}0.1253 \\
(0.168) \\
\end{array}$ \\
\hline Weibull & $\begin{array}{l}0.180 \\
(0.040)\end{array}$ & $\begin{array}{l}1.147 \\
(0.101) \\
\end{array}$ & & & & 0.2424 & 1.4901 & $\begin{array}{l}0.1159 \\
(0.239) \\
\end{array}$ \\
\hline $\begin{array}{l}\text { Inverse } \\
\text { Weibull }\end{array}$ & $\begin{array}{l}1.797 \\
(0.203)\end{array}$ & $\begin{array}{l}1.126 \\
(0.098) \\
\end{array}$ & & & & 0.2745 & 1.6926 & $\begin{array}{l}0.1253 \\
(0.168) \\
\end{array}$ \\
\hline
\end{tabular}

Table 8: Goodness-of-fit statistics for data set III

\begin{tabular}{|l|r|r|r|r|r|}
\hline Model & \multicolumn{1}{|l|}{ AIC } & \multicolumn{1}{l|}{ CAIC } & \multicolumn{1}{l|}{ BIC } & \multicolumn{1}{l|}{ HQIC } & \multicolumn{1}{l|}{$\ell$} \\
\hline MBXII- Power & 357.59 & 358.14 & 367.02 & 361.37 & 174.80 \\
\hline BXII- Power & 374.25 & 374.58 & 381.32 & 377.08 & 184.13 \\
\hline L- Power & 378.55 & 378.71 & 383.26 & 380.44 & 187.28 \\
\hline LL- Power & 378.27 & 378.43 & 382.98 & 380.16 & 187.13 \\
\hline Power & 395.82 & 395.88 & 398.18 & 396.77 & 196.91 \\
\hline W-Power & 375.37 & 375.70 & 382.44 & 378.20 & 184.69 \\
\hline MBXII & 389.79 & 390.11 & 396.89 & 392.63 & 191.89 \\
\hline MBIII & 391.78 & 392.1 & 398.89 & 394.63 & 192.89 \\
\hline Weibull & 387.79 & 387.94 & 392.52 & 389.68 & 191.89 \\
\hline Inverse Weibull & 389.78 & 389.94 & 394.52 & 391.68 & 192.89 \\
\hline
\end{tabular}

The MBXII-Power distribution is best fitted model than the other sub-models because the values of all criteria of goodness of fit are significantly smaller for the MBXII-Power distribution. 

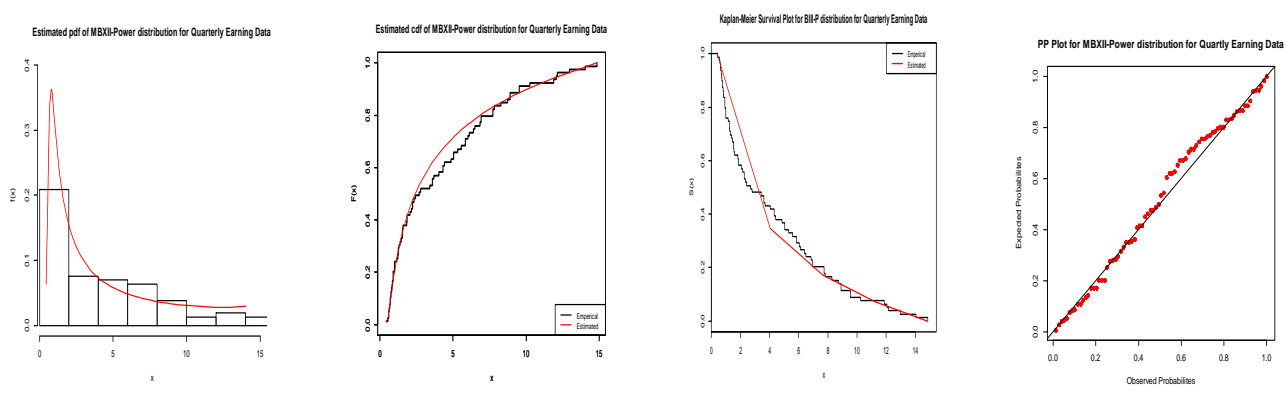

Fig. 7: Fitted pdf, cdf, survival and pp plots of the MBXII-Power distribution for Quarterly earnings data

We can observe that the MBXII-Power distribution is close to empirical data (Fig.7).

\section{CONCLUDING REMARKS}

We have developed the MBXII-Power distribution along with properties such as submodels, moments, inequality measures, residual and reverse residual life function, stressstrength reliability and multicomponent stress-strength reliability model. The MBXIIPower distribution is characterized via different techniques. Maximum Likelihood estimates are computed. The simulation study is performed on the basis of graphical results by using the MBXII-Power distribution to see the performance of MLEs. Goodness of fit show that the MBXII-Power distribution is a better fit. Applications of the MBXII-Power model to survival times of pigs, survival times of patients and quarterly earnings are presented to show its significance and flexibility. We have proved that the MBXII-Power distribution is empirically better for survival times of pigs, survival times of patients and quarterly earnings.

\section{REFERENCES}

1. Afify, A. Z., Cordeiro, G. M., Ortega, E. M., Yousof, H. M., \& Butt, N. S. (2018). The four-parameter Burr XII distribution: Properties, regression model, and applications. Communications in Statistics-Theory and Methods, 47(11), 26052624.

2. Alzaatreh, A., Lee, C., and Famoye, F. (2013). A new method for generating families of continuous distributions. Metron, 71(1), 63-79.

3. Arnold, B.C., \& Balakrishnan, N., and Nagarajah, H. (1998). Records, John Wiley, New York.

4. Athar, H., \& Noor, Z. (2014). Characterization of probability distributions by conditional expectations of functions of record statistics, Journal of the Egyptian Mathematical Society, 22, 275-279.

5. Bhattacharyya, G. K., \& Johnson, R. A. (1974). Estimation of reliability in a multicomponent stress-strength model. Journal of the American Statistical Association, 69(348), 966-970.

6. Bhatti, F. A., Ali, A., Hamedani, G. G., \& Ahmad, M. (2018). On Generalized Log Burr Xii Distribution. Pakistan Journal of Statistics and Operation Research, 14(3), 615-643. 
7. Bhatti, F. A., Hamedani, G. G., \& Ahmad, M. (2018). On Modified Log Burr XII Distribution. JIRSS-Journal Of The Iranian Statistical Society, 17(2), 57-89.

8. Bourguignon, M., Silva, R.B. and Cordeiro, G.M.(2014). The Weibull-G Family of Probability Distributions. Journal of Data Science, 12, 53-68.

9. Burr, I.W. (1942). Cumulative frequency functions. The Annals of Mathematical Statistics, 13(2), 215-232.

10. Feigl, P. and Zelen, M. (1965). Estimation of exponential survival probabilities with concomitant information. Biometrics, 826-838.

11. Ghosh, I., \& Bourguignon, M. (2017). A New Extended Burr XII Distribution. Austrian Journal of Statistics, 46(1), 33-39.

12. Glänzel, W. A. (1990). Some consequences of a characterization theorem based on truncated moments, Statistics 21 (1990) ; 613 - 618:

13. Gomes, A. E., da-Silva, C. Q., \& Cordeiro, G. M. (2015). Two extended Burr models: Theory and practice. Communications in Statistics-Theory and Methods, 44(8), 1706-1734.

14. Guerra, R. R., Peña-Ramírez, F. A., \& Cordeiro, G. M. (2017). The gamma Burr XII distribution: Theory and application. Journal of Data Science, 15(3).

15. Gurvich, M. R., Dibenedetto, A. T., \& Ranade, S. V. (1997). A new statistical distribution for characterizing the random strength of brittle materials. Journal of Materials Science, 32(10), 2559-2564.

16. Hamedani, G. G. (2013). On certain generalized gamma convolution distributions II. Technical Report, 484, MSCS, Marquette University.

17. Khan, A.H., and Alzaid, A.A. (2004). Characterization of distributions through linear regression of non-adjacent generalized order statistics, Journal of Applied Statistical Science, 13, 123-136.

18. Mdlongwa, P., Oluyede, B., Amey, A., \& Huang, S. (2017). The Burr XII modified Weibull distribution: model, properties and applications. Electronic Journal of Applied Statistical Analysis, 10(1), 118-145.

19. Mead, M. E., \& Afify, A. Z. (2017). On five-parameter Burr XII distribution: properties and applications. South African Statist. J, 51, 67-80.

20. Muhammad, M. (2016). A generalization of the Burr XII-Poisson distribution and its applications. Journal of Statistics Applications \& Probability, 5(1), 29-41.

21. Nagarajah, H. (1988). Some characterizations of continuous distributions based on regressions of adjacent order statistics and record values, Sankhya Series A., 50, 70-73.

22. Olapade, A.K. (2008). On a six-parameter generalized Burr XII distribution, Electronic Journal of Statistics Mathematical Statistics, pp.1-5.

23. Paranaíba, P.F., Ortega, E.M., Cordeiro, G.M. and Pescim, R.R. (2011). The beta Burr XII distribution with application to lifetime data. Computational Statistics \& Data Analysis, 55(2), 1118-1136.

24. Paranaíba, P.F., Ortega, E.M., Cordeiro, G.M. and Pascoa, M.A.D. (2013). The Kumaraswamy Burr XII distribution: theory and practice. Journal of Statistical Computation and Simulation, 83(11), 2117-2143.

25. Shao, Q., Wong, H., Xia, J. and Ip, W.C. (2004). Models for extremes using the extended three-parameter Burr XII system with application to flood frequency analysis. Hydrological Sciences Journal, 49(4), 685-701. 
26. Silva, R.B. and Cordeiro, G.M. (2015). The Burr XII power series distributions: A new compounding family. Brazilian Journal of Probability and Statistics, 29(3), 565-589.

27. Tahir, M., Alizadeh, M., Mansoor, M., Cordeiro, G. M., and Zubair, M. (2014). The Weibull-power function distribution with applications. Hacettepe university bulletin of natural sciences and engineering series $b$ : mathematics and statistics

28. Takahasi, K. (1965). Note on the Multivariate Burr's Distribution. Annals of the Institute of Statistical Mathematics, 17, 257-260.

Technology, and Sciences (ASRJETS), 26(2), 18-32.

29. Yari, G., \& Tondpour, Z. (2017). The new Burr distribution and its application. Mathematical Sciences, 11(1), 47-54.

\section{Lemma. (i)}

\section{APPENDIX A}

If $Z_{1} \sim \exp$.(1) and $Z_{2} \sim \operatorname{Gamma}(\alpha / \gamma, 1)$, then for $Z_{1}=\gamma\left(\frac{X^{\kappa}}{\lambda^{\kappa}-X^{\kappa}}\right)^{\beta} Z_{2}$, we have

$$
X=\lambda\left[1+\left(\gamma Z_{2}\left(Z_{1}\right)^{-1}\right)^{\frac{1}{\beta}}\right]^{-\frac{1}{\kappa}} \sim \text { MBXII - Power }(\alpha, \beta, \gamma, \kappa, \lambda) .
$$

\section{Proof}

The pdf for $Z_{1} \sim \exp .(1)$ is $f\left(z_{1}\right)=e^{-z_{1}}, z_{1}>0$.

The pdf for $Z_{2} \sim \operatorname{Gamma}(\alpha / \gamma, 1)$, is $f\left(z_{2}\right)=\frac{z_{2}^{\frac{\alpha}{\gamma}-1} e^{-z_{2}}}{\Gamma(\alpha / \gamma)}, z_{2}>0$.

The joint distribution of both random variables is $f\left(z_{1}, z_{2}\right)=\frac{z_{2}^{\frac{\alpha}{\gamma}-1} e^{-z_{2}} e^{-z_{1}}}{\Gamma(\alpha / \gamma)}, z_{1}>0, z_{2}>0$.

Let $Z_{1}=\gamma\left(\frac{X^{\kappa}}{\lambda^{\kappa}-X^{\kappa}}\right)^{\beta} Z_{2}$, then Jacobean is $|J|=\gamma \beta \frac{\kappa \lambda^{\kappa} x^{\kappa-1}}{\left(\lambda^{\kappa}-x^{\kappa}\right)^{2}}\left(\frac{x^{\kappa}}{\lambda^{\kappa}-x^{\kappa}}\right)^{\beta-1} z_{2}$.

The joint distribution of both random variables $X$ and $Z_{2}$ is

$$
f\left(x, \mathrm{z}_{2}\right)=\frac{\left(z_{2}\right)^{\frac{\alpha}{\gamma}-1} e^{-z_{2}} e^{-\gamma\left(\frac{x^{\kappa}}{\lambda^{\kappa}-x^{\kappa}}\right)^{\beta} z_{2}}}{\Gamma(\alpha / \gamma)} \gamma \beta \frac{\kappa \lambda^{\kappa} x^{\kappa-1}}{\left(\lambda^{\kappa}-x^{\kappa}\right)^{2}}\left(\frac{x^{\kappa}}{\lambda^{\kappa}-x^{\kappa}}\right)^{\beta-1} z_{2}, \quad x>0, z_{2}>0
$$

The pdf of the random variables $X$ is

$$
f(x)=\frac{\alpha \beta \kappa \lambda^{\kappa} x^{\beta \kappa-1}}{\left(\lambda^{\kappa}-x^{\kappa}\right)^{\beta+1}}\left[1+\gamma\left(\frac{x^{\kappa}}{\lambda^{\kappa}-x^{\kappa}}\right)^{\beta}\right]^{-\frac{\alpha}{\gamma}-1}, 0<x<\lambda
$$

